\title{
New asymptotic giant branch models for a range of metallicities ${ }^{\star}$
}

\author{
A. Weiss ${ }^{1}$ and J. W. Ferguson ${ }^{2,1}$ \\ 1 Max-Planck-Institut für Astrophysik, Karl-Schwarzschild-Str. 1, 85748 Garching, Germany \\ e-mail: weiss@mpa-garching.mpg.de \\ 2 Physics Department, Wichita State University, Wichita, KS 67260-0032, USA
}

Received 12 March 2009 / Accepted 14 July 2009

\begin{abstract}
We present a new grid of stellar model calculations for stars on the Asymptotic Giant Branch between 1.0 and $6.0 M_{\odot}$. Our grid consists of 10 chemical mixtures with 5 metallicities between $Z=0.0005$ and $Z=0.04$, and with both solar-like and $\alpha$-element enhanced metal ratios for each metallicity. We treat consistently the carbon-enhancement of the stellar envelopes by using opacity tables with varying $\mathrm{C} / \mathrm{O}$-ratio and by employing theoretical mass loss rates for carbon stars. The low temperature opacities have been calculated specifically for this project. For oxygen stars we use an empirical mass loss formalism. The third dredge-up is naturally obtained by including convective overshooting. Our models reach effective temperatures in agreement with earlier synthetic models, which included approximative carbon-enriched molecular opacities and show good agreement with empirically determined carbonstar lifetimes. A fraction of the models could be followed into the post-AGB phase, for which we provide models in a mass range supplementing previous post-AGB calculations. Our grid constitutes the most extensive set of AGB-models, calculated with the latest physical input data and treating carbon-enhancement due to the third dredge-up most consistently.
\end{abstract}

Key words. stars: evolution - stars: AGB and post-AGB - stars: interiors

\section{Introduction}

A number of stellar model libraries have been developed to serve as databases for applications in various fields of astrophysics. These range from fitting evolutionary tracks to individual stars, to isochrone matching for stellar clusters, up to complete population syntheses of galaxies. The latter purpose is probably the most frequent one. Examples for such libraries are those at Padova (Girardi et al. 2000, and references therein), BaSTI at Teramo (Pietrinferni et al. 2004), and most recently the one at Dartmouth College (Dotter et al. 2008). These libraries are constantly updated by new calculations and extended by covering more and more chemical compositions.

In terms of the evolutionary phases covered, all of the just cited libraries provide the results of full stellar models of low and intermediate mass $\left(1 \lesssim M / M_{\odot} \lesssim 8\right)$ up to the onset of thermal pulses (TP) on the asymptotic giant branch (AGB). One reason is the prohibitive effort to follow the TP-AGB phase with complete models, not to speak of the notorious numerical problems encountered during this phase of strongly changing timescales that can be as short as hours during a helium shell flashing. Another reason not to include full models in the libraries is the uncertainty of AGB calculations, owing to the importance of rather ill-known physical effects, such as overshooting, rotation, and mass loss (see Herwig 2005, for a review of the current state of the art problems).

However, for populations with an age of a few hundred million to about 2 billion years, the contribution of intermediate mass stars cannot be ignored. Because of their high luminosity they contribute significantly to the integrated light, and due to their low surface temperatures they dominate the spectra and

^ Appendices are only available in electronic form at http://www . aanda.org colours in the near infrared. Liu et al. (2000), Raimondo et al. (2005) and others have demonstrated this effect convincingly. The Padova and BaSTI stellar model libraries have included the TP-AGB recently (Marigo \& Girardi 2007; Cordier et al. 2007) by making use of synthetic AGB-models (for a selection of historical and modern synthetic AGB-models see Iben \& Truran 1978; Renzini \& Voli 1981; Groenewegen \& de Jong 1993; Marigo et al. 1996). Synthetic AGB-models try to predict basic stellar parameters, such as $L(t), T_{\text {eff }}(t)$ and stellar yields without resorting to calculations of full models, but use relationships obtained from full calculations. However, they are by no means merely reproducing the full models. Rather, they are using basic properties, such as the mass of the helium core or the luminosity of the helium shell as function of time as input for calculations of mass loss, effective temperature, envelope composition and even nucleosynthesis at the bottom of the convective envelope (the Hot Bottom Burning; HBB). The idea is to take the complicated core and helium shell evolution from full models and add the evolution of the hydrogen layers, which is less difficult to compute by on-line calculations. With this approach the synthetic models can treat effects like the third-dredge up or mass loss as an additional, free-to-chose property, which is then usually calibrated by comparison of the synthetic models with observed AGB-star samples.

To some extent all synthetic models depend on results from full stellar modelling, and therefore extensive computations of the evolution along the AGB are needed. The most widely used AGB- and post-AGB tracks are those by Vassiliadis \& Wood (1993, 1994) and Bloecker (1995b,a). The former calculations were done for initial masses between 0.89 and $5 M_{\odot}$, and for chemical compositions of $Z=0.016$ ("solar"), 0.008 ("LMC"), 0.004 ("SMC"), and 0.001 ("Pop. II"). However, not all the mass values were calculated for each metallicity. The most metal-poor 
Table 1. Initial compositions used for the model calculations.

\begin{tabular}{|c|c|c|c|c|c|}
\hline Metallicity & Super-solar & Solar & $\overline{\mathrm{LMC}}$ & SMC & Metal poor \\
\hline$Z$ & 0.04 & 0.02 & 0.008 & 0.004 & 0.0005 \\
\hline$X$ & 0.635 & 0.695 & 0.731 & 0.743 & 0.7535 \\
\hline$Y$ & 0.325 & 0.285 & 0.261 & 0.253 & 0.2460 \\
\hline Met. scaling & $\alpha$-enh. & $\alpha$-enh. & $\alpha$-enh. & $\alpha$-enh. & $\alpha$-enh. \\
\hline mixture & II & III & VI & VIII & IX \\
\hline
\end{tabular}

set consists of only the 1.0 and $1.5 M_{\odot}$ model. Blöcker calculated models for only a Pop. I metallicity, $Z=0.021\left(1 \leq M / M_{\odot}<7\right)$. Wagenhuber \& Groenewegen (1998) have provided fitting functions for synthetic populations based on the set of AGB calculations by Wagenhuber (1996) for three metallicities $(Z=0.02$, 0.008 , and 0.001 ) and masses from 1 to $7 M_{\odot}$. Most recently, a new comprehensive set has been added by Karakas (2003) for $1 \leq M / M_{\odot} \leq 6$ and $Z=0.004,0.008$, and 0.02 , although the intention of this work has been to follow the nucleosynthesis in AGB stars, and not to provide a grid of AGB models for general usage. Obviously, the emphasis in all work quoted has been to provide models adequate for solar-type stars and for the Large and Small Magellanic Clouds, from which we have the largest amount of information about this evolutionary phase. For use in population synthesis models of galaxies, this range of model compositions is certainly not sufficient.

Many of the physical ingredients for the models have been improved, in part considerably, since the generation of all these grids of AGB and post-AGB models. Most importantly, the available models were calculated using opacities and equation of state prior than those by the OPAL group (Iglesias \& Rogers 1996; Rogers et al. 1996) or by the Opacity Project (Seaton 2007), which rests on the "MHD" equation of state (Mihalas et al. 1988). Only in Karakas (2003) high-temperature OPAL opacities were used. Similarly, the nuclear reactions and neutrino emission rates for most grids are from the ' 80 s or are even older in some cases.

It is therefore timely to provide new grids for AGB stars, which include both the latest physical ingredients, and for a larger variety of chemical compositions. Section 2 presents the general structure of the stellar evolution program used. In Sect. 3 the details of the code specific to this project, which constitute the improvement over previous grids for AGB models will be discussed in detail. Apart from the aspect of updated constitutional physics and more extensive chemical compositions our emphasis lies on a consistent treatment of carbon enrichment of the envelope due to the third dredge-up. This includes the influence on the opacities, which has been shown by Marigo (2002) to be crucial for the stars' temperature. This, in turn, is the most important parameter for dust-driven mass loss, which dominates the late AGB evolution and the transition to the postAGB. In Sect. 4 the results of our calculations will be presented. Conclusions will close the paper in Sect. 5.

\section{Program and calculation set-up}

\subsection{Chemical composition and stellar mass grid}

In addition to the previously mentioned chemical compositions representative for galactic Population I ("solar"), the LMC and SMC stars, we have added one super-solar composition with $Z=0.04$ and one metal-poor with $Z=0.0005$. For the helium content of each mixture we used

$Y=Y_{\mathrm{p}}+Z \times \frac{\Delta Y}{\Delta Z}$
Table 2. Logarithmic element abundances within the metal group.

\begin{tabular}{rcc}
\hline \hline El. $^{a}$ & Solar $^{b}$ & $\alpha$-enhanced \\
\hline $\mathrm{C}$ & 8.55 & 8.55 \\
$\mathrm{~N}$ & 7.97 & 7.97 \\
${ }^{*} \mathrm{O}$ & 8.87 & 9.27 \\
${ }^{*} \mathrm{Ne}$ & 8.07 & 8.47 \\
$\mathrm{Na}$ & 6.33 & 6.33 \\
${ }^{*} \mathrm{Mg}$ & 7.58 & 7.98 \\
$\mathrm{Al}$ & 6.47 & 6.47 \\
${ }^{*} \mathrm{Si}$ & 7.55 & 7.95 \\
$\mathrm{P}$ & 5.45 & 5.45 \\
${ }^{*} \mathrm{~S}$ & 7.21 & 7.61 \\
$\mathrm{Cl}$ & 5.50 & 5.50 \\
$\mathrm{Ar}$ & 6.52 & 6.52 \\
$\mathrm{~K}$ & 5.12 & 5.12 \\
${ }^{*} \mathrm{Ca}$ & 6.36 & 6.76 \\
${ }^{*} \mathrm{Ti}$ & 5.02 & 5.42 \\
$\mathrm{Cr}$ & 5.67 & 5.67 \\
$\mathrm{Mn}$ & 5.39 & 5.39 \\
$\mathrm{Fe}$ & 7.51 & 7.51 \\
$\mathrm{Ni}$ & 6.25 & 6.25 \\
\hline
\end{tabular}

${ }^{a} \alpha$-elements are marked with an asterisk; ${ }^{b}$ Seaton et al. (1992); Grevesse \& Noels (1993); ${ }^{c}$ an $\alpha$-enhancement of +0.4 dex was assumed.

with $Y_{\mathrm{p}}=0.245$ for the primordial helium content and $\Delta Y / \Delta Z=$ 2.0 for the connection between helium and metal production. The first value is in agreement with the cosmological model and was also used, for example, by Cordier et al. (2007), the second one is within the general range of determination. In addition, for the first time $\alpha$-element enhancement was taken into consideration in an AGB model grid, since the ratio of elements of the $\alpha$-group relative to those of the iron-peak depends on the star formation history, which might differ radically from that of the galactic disk and halo. Examples are the galactic bulge and elliptical galaxies, where solar- and super-solar iron abundances along with $\alpha$-enhancement are found. For each metallicity listed above, both a solar-scaled and an $\alpha$-enhanced metal mixture was used. In Tables 1 and 2 we list ten mixtures for which we are providing models and the element distributions within the "metals". The solar mixture is that of Seaton et al. (1992), which is almost identical to that of Grevesse \& Noels (1993); the $\alpha$ enhancement is simply taken as an additional +0.4 dex for all respective elements. These metal mixtures are identical to those in Coelho et al. (2007).

For all 10 chemical compositions 11 initial mass values between 1 and $6 M_{\odot}$ were calculated. They are 1.0, 1.2, 1.5, 1.6, $1.8,2.0,2.6,3.0,4.0,5.0$, and $6.0 M_{\odot}$. Calculations were started on the zero-age main-sequence (ZAMS) and continued as far as possible with the aim of reaching the white dwarf cooling track. 


\subsection{Stellar evolution program: basic properties}

For all calculations the Garching Stellar Evolution Code (GARSTEC) as described most recently in Weiss \& Schlattl (2008) was used. Changes specifically applied for the present calculations will be discussed in the following section. The program is able to produce an up-to-date standard solar model (Weiss \& Schlattl 2008), from which a mixing length parameter of $\alpha_{\mathrm{MLT}}=1.74$ is obtained. For the calculations presented here a value of 1.75 was used. The slight difference stems from the use of the solar metal distribution of Grevesse \& Sauval (1998) in Weiss \& Schlattl (2008).

GARSTEC is able to follow low-mass stars through the core helium flash (e.g. Meissner \& Weiss 2006) and many thermal pulses on the AGB without human intervention since the numerical improvements by Wagenhuber \& Weiss (1994). Nevertheless, towards the end of the TP-AGB phase convergence problems still exist and inhibit a continuous modeling of the whole stellar evolution. We will return to this problem in Sect. 4.2.1.

Although particle diffusion is implemented in the program, and used in the solar model calibration, the present calculations were done without employing it. However, a diffusive scheme is used for convective mixing. Mixing and nuclear burning are solved simultaneously in one set of equations, which is of particular importance for fast nuclear burning phases, as in case of mixing of protons into hot carbon-helium-layers. This may happen during the core helium flash in extremely metal-poor stars (Schlattl et al. 2001) or in the TP-AGB phase.

Since we were interested in the structural properties of AGB stars, and not in their chemical yields, the nuclear network was restricted to standard hydrogen and helium burning reactions. If needed, both burning phases can be solved together. The reaction rates are mainly from Caughlan et al. (1985) and Adelberger et al. (1998), with the following updates: We use the $3 \alpha$-rate by Fynbo et al. (2005), the rate for the CNO bottleneck reaction ${ }^{14} \mathrm{~N}(\mathrm{p}, \gamma){ }^{16} \mathrm{O}$ by Formicola et al. (2004), and the ${ }^{12} \mathrm{C}(\alpha, \gamma){ }^{16} \mathrm{O}$ rate by Kunz et al. (2002). The influence of the former two updates was discussed already in Weiss et al. (2005). A deficit of this restricted nuclear reaction network is that the hotter protoncycles as well as $\alpha$-captures on intermediate elements such as nitrogen and oxygen are missing. Therefore the abundance of nitrogen during the TP-AGB phase is always taken to be an upper limit, because part of it could be processed into heavier elements by such reactions.

The equation of state is the FreeEOS ${ }^{1}$ of $\mathrm{A}$. Irwin (see Cassisi et al. 2003). We use Eddington grey atmospheres. All further details about our stellar evolution code can be found in Weiss \& Schlattl (2008).

\section{Code improvements for AGB modeling}

The evolution along the AGB is characterized by the internal nuclear processes, leading to increasing luminosities and larger stellar radii, and by the strong mass loss due to stellar winds, which depend on mass, radius, luminosity, and chemical composition of the envelope. As is known from the initial-mass to final-mass relation (see Weidemann 2000, for a comprehensive overview and Sect. 4.2.4), the wide range of initial masses $\left(1 \lesssim M / M_{\odot} \lesssim 8\right)$ results - due to the overwhelming effect of strong mass loss at higher AGB-luminosities - in quite a narrow range of white dwarf masses $\left(0.5 \lesssim M / M_{\odot} \lesssim 1.1\right)$. The mass loss

\footnotetext{
${ }^{1}$ Available at http://freeeos.sourceforge.net
}

itself depends on the chemical composition of the atmosphere and envelope, which itself is modified by internal nuclear processes and mixing between the convective envelope and regions of nuclear burning. Notably the third dredge-up, which leads to the enrichment of the envelope with carbon from the heliumburning layers, and which is the result of structure changes in the course of the thermal pulses of the helium shell, is ultimately linked to the mass loss. The enrichment of carbon in the outer stellar layers allows the formation of carbon-molecules and dust, which then leads to dust-driven winds. Dust formation and wind depend strongly on stellar temperatures, which itself is determined by the superadiabatic convection of the envelope. The effectiveness of this convection depends upon the radiative transport and opacites which in turn are a function of the carbon abundance. It is the aim of our calculations to treat the carbon abundance variations of the stellar envelope and the consequences of it as consistently as possible. This implies a detailed treatment of nuclear processes, mixing, opacities, and mass loss. In the following we will present how we approach this problem. The nuclear processes (carbon production during helium burning, $\mathrm{CNO}$ equilibrium) were previously discused in the preceeding section.

\subsection{Dredge-up by convective overshooting}

In AGB-calculations with GARSTEC, as with most other programs, the third dredge-up does not occur with the canonical physics described so far. If the third dredge-up occurs in our models it would show only for higher masses at high AGBluminosities (core masses) and low metallicity (Wagenhuber 1996). This is in contradiction to observations and therefore additional mixing mechanisms are needed to ensure the appearance of carbon stars on the lower AGB for all populations. Several such processes have been invoked, such as gravity waves (Denissenkov \& Tout 2003), rotationally induced mixing (Langer et al. 1999), or convective overshooting (Herwig et al. 1997). We adopt the latter approach here, but keep in mind that overshooting could be just representative for the combined effect of several physical processes acting simultaneously. In synthetic models the amount of dredged-up material is adjusted to reproduce observed carbon-star statistics.

The physical concept of overshooting we have implemented is that of an exponentially decaying velocity field outside regions formally convective according to the Schwarzschild-criterion (Freytag et al. 1996). This is cast into a diffusion equation

$$
D_{\mathrm{ov}}=v_{0} \cdot H_{\mathrm{P}}(0) \exp \left[\frac{-2 z}{f \cdot H_{\mathrm{P}}(0)}\right]
$$

where $z$ is the distance from the boundary in the outer radiative region, $H_{P}(0)$ is the pressure scale height taken at the boundary of the convectively unstable region, i.e. at $z=0$, and $v_{0}$ the typical velocity of the convective elements (obtained from mixing length theory) at the inner side of the Schwarzschild border, following Herwig et al. (1997). $f$ is a free overshooting parameter. It represents a measure of the efficiency of the extra mixing. The larger the value of $f$, the further the extra mixing extends outside the convective region.

The same approach has already been used, for example, by Herwig $(2004 b$,a) for AGB models. The value for the parameter $f$ initially used in Herwig et al. (1997) was $f=$ 0.02 - based on earlier main-sequence width fitting - to obtain sufficient third dredge-up. These authors stated that qualitatively their result do not change if $f$ varies within a factor of 2. A somewhat smaller value of $f=0.016$ was used by 
Herwig et al. (1999) and repeatedly in later papers (Herwig 2000, 2004b,a). However, detailed investigations into the nucleosynthetic products of AGB-evolution with overshooting complicated the picture. While Herwig et al. (1999) emphasize the need for $f \approx 0.016$ for the overshooting at the bottom of the pulse-driven convective layer, in order to reproduce the abundance patterns of post-AGB stars of type PG1159, Lugaro et al. (2003) argue for a lower efficiency at the convective border (they suggest a value of 0.008) to achieve better agreement with detailed $s$-process abundance patterns. To further complicate issues, a recent 3-dimensional hydrodynamical study by Herwig et al. (2007) indicates a varying effective $f$ at the bottom of the pulse-driven convection zone between 0.01 and 0.14 .

Similarly, to achieve sufficient efficiency of the ${ }^{13} \mathrm{C}$ neutron source in low-mass stars, Lugaro et al. (2003) used $f=0.128$ for overshooting from the bottom of the convective envelope, while Herwig (2004a) finds that a value of 0.03 prematurely stops the AGB evolution of a $5 M_{\odot}$ star. One should note that these detailed investigations were done for either a single or a few stellar models, therefore the results cannot be generalized to all masses and metallicities.

In conclusion, given the unclear situation about the extent of overshooting at the lower boundary of the pulse-driven convection zone, and the finding that the overall evolution does not change dramatically if $f$ is varied within a factor of 2 , we chose to employ one value for $f$ at all convective boundaries. This value is $f=0.016$, which is the "generic" value by Herwig. For a slightly higher value of 0.018 we achieved good fits to galactic open clusters colour-magnitude diagrams (unpublished). We are aware that our value might be too high for the lower boundary of the pulse-driven convection zone; a conclusion recently strengthened by Salaris et al. (2009) from implications of the initial-final-mass relation. We will return to this in Sect. 4.2.4. Our choice is also in agreement with Miller Bertolami \& Althaus (2006a).

The only exception from our procedure is overshooting from convective cores on the main sequence, where overshooting is restricted for small convective core sizes, in agreement with similar approaches found in, for example, Ventura et al. (1998). For $1.0 \leq M / M_{\odot}<1.5$ the overshooting efficiency is gradually increased: starting from a value of 0 for $M_{\text {ZAMS }}=1.0 M_{\odot}$ it reaches 0.016 at $M_{\text {ZAMS }}=1.5 M_{\odot}$. The intermediate values are given by the relation $f=0.032 \cdot\left(M / M_{\odot}-1.0\right)$.

Figure 1 shows the effect of the third dredge-up on the abundances of $\mathrm{C}, \mathrm{N}$, and $\mathrm{O}$ and the $\mathrm{C} / \mathrm{O}$-ratio for a model of $2.6 \mathrm{M}_{\odot}$ with a solar-like metal abundance of 0.02 (mix III of Table 1) as caused by our overshooting description. This model does not experience HBB. Dredge-up starts after the 6th of 14 thermal pulses. Note that oxygen is enhanced, too, due to the third dredge-up.

\subsection{Opacities for carbon-enriched compositions}

The carbon (and partially oxygen) enrichment of the envelope due to the third dredge-up has to be reflected in the treatment of the constitutional physics of the models. Where element abundances appear explicitly, as in the nuclear reactions, this is trivial. The use of tables for the equation of state and the Rosseland opacity inhibits this direct approach, however. For the equation of state, composition changes within the metal-group are not taken into account; generally, one assumes that due to the low abundance of individual metals, even after dredge-up, the equation of state is sufficiently accurate if the total metallicity $Z$ is

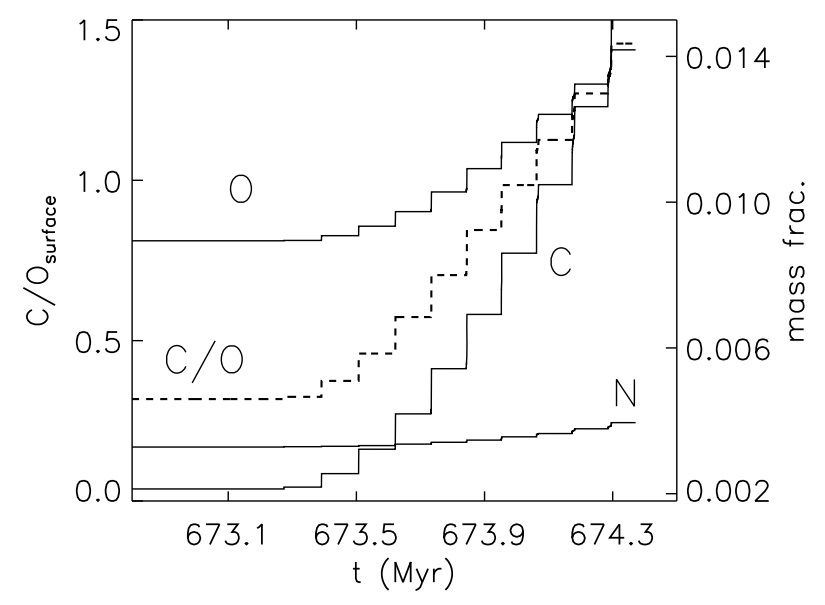

Fig. 1. $\mathrm{C}, \mathrm{N}$, and $\mathrm{O}$ mass fractions (right axis) in an $2.6 M_{\odot} \operatorname{model}(Z=$ 0.02; solar metal ratios) during TP-AGB model as a consequence of the third dredge-up. The dashed line corresponds to the C/O-ratio (left axis).

taken into account properly, which is the case in our calculations.

The situation is different for opacities, where the absorption properties can be more important than the absolute abundance of an absorber. Marigo (2002) has convincingly shown that the outer envelope structure of AGB stars depends considerably on the opacities, and that in particular carbon-enriched molecular opacities reduce effective temperatures significantly, leading to much better agreement of colours of synthetic populations with observations.

Her adopted procedure to compute the molecular opacities, through analytical fit relations, closely resembles that of Scalo \& Ulrich (1975) and is incorporated in the P. Marigo synthetic code for TP-AGB evolution, and in the most recent models of the Padova stellar model library (Marigo \& Girardi 2007). The possibility to consistently compute the opacities for any chemical composition, during the evolutionary calculations is a huge advantage of this approach and the effects detected in the models help to account for a number of observational properties of carbon stars. However, this has never been implemented in full stellar models, with the exception of the recent and independent work by Cristallo et al. (2007), who used the molecular opacities of Lederer \& Aringer (2008), but presented results solely for one single model $\left(2 M_{\odot} ; Z=0.0001\right)$. Recently, more $2 M_{\odot}$ models for additional metallicities became available (Cristallo et al. 2009).

\subsubsection{WSU tables for molecular opacities}

For our models new opacity tables have been prepared for C-enhanced mixtures. For high temperatures, OPAL-tables for atomic opacities (Iglesias \& Rogers 1996) were obtained from the OPAL-website ${ }^{2}$, and for low temperatures new tables for molecular opacities were specifically generated with the method and program described in Ferguson et al. (2005), in the following called WSU (Wichita State University) tables. In all cases the chemical compositions of low- and high-T tables agree and tables from the different sources are combined as described in Weiss \& Schlattl (2008).

For the absorption properties it is necessary to know which molecules are present in cool stellar matter. A crucial

2 http://physci.llnl.gov/Research/OPAL 


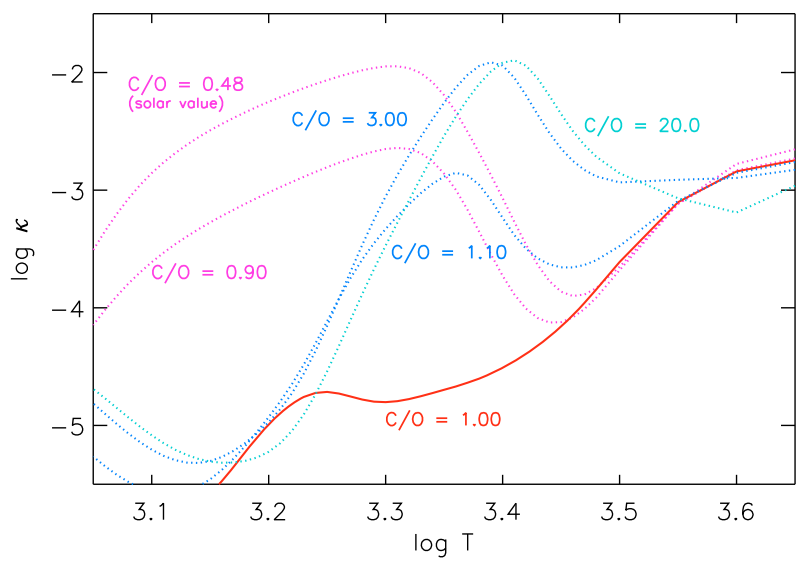

Fig. 2. Rosseland mean opacity $\kappa$ as function of temperature for a mixture with $X=0.7, Z=0.02$ and a value of $\log R=\log \rho-3 \log T+18=$ -2.0 . The different lines are for the various $\mathrm{C} / \mathrm{O}$-ratios of the mixtures given in Table 3 .

quantity is the $\mathrm{C} / \mathrm{O}$-ratio, because of the high binding energy of the $\mathrm{CO}$-molecule. If the $\mathrm{C} / \mathrm{O}$ ratio is less than $\approx 1$ (oxygen-stars of type $\mathrm{M}$ or $\mathrm{S}$ ), stellar spectra show strong absorption bands of $\mathrm{TiO}, \mathrm{VO}$, and $\mathrm{H}_{2} \mathrm{O}$. In the other case (carbon-stars of type $\mathrm{C}$ or $\mathrm{R}$ and $\mathrm{N}$ ), basically all $\mathrm{O}$ is bound in $\mathrm{CO}$ with $\mathrm{CN}, \mathrm{C}_{2}$ and $\mathrm{SiC}$ and some $\mathrm{HCN}$ and $\mathrm{C}_{2} \mathrm{H}_{2}$ formed from the remaining carbon. Thus, the $\mathrm{C} / \mathrm{O}$-ratio is more important than the absolute carbon abundance in the stellar mixture and therefore the additional opacity tables were produced as function of varying $\mathrm{C} / \mathrm{O}$-ratio (for each choice of $X, Z$, and $\alpha$-abundance), but not of absolute carbon abundance. For the computations presented here the table values of $\mathrm{C} / \mathrm{O}$ were 0.48 (solar value), $0.9,1.0,1.1,3.0$, and 20. This choice was guided by an investigation of the change in the Rosseland mean opacity due to variations of $\mathrm{C} / \mathrm{O}$.

Figure 2 shows how $\kappa$ changes with varying $\mathrm{C} / \mathrm{O}$ ratio. The most sensitive regime is around $\mathrm{C} / \mathrm{O} \approx 1$, while for $\mathrm{C} / \mathrm{O}>3$ hardly any change in the mean opacity is apparent. The temperature range significant for AGB models is approximately $\log T \geq 3.3$, which justifies our grid of C/O-ratios. Ferguson \& Dotter (2008) discuss in detail some of the important features of Fig. 2. An important point is that as the $\mathrm{C} / \mathrm{O}$ ratio increases the amount of $\mathrm{O}$ available for molecular $\mathrm{H}_{2} \mathrm{O}$ is decreased (becomes locked in $\mathrm{CO}$ ) thus decreasing the mean opacity at temperatures important for $\mathrm{H}_{2} \mathrm{O}$ absorption. At $\mathrm{C} / \mathrm{O} \approx 1$, most of the molecular opacity is in molecular $\mathrm{CO}$, a low absorber. At higher values of $\mathrm{C} / \mathrm{O}$ the opacity becomes dominated by $\mathrm{CN}$.

Recently, Lederer \& Aringer (2009) published similar opacity data for chemical mixtures with varying $\mathrm{C}$ and $\mathrm{N}$ abundances, which show similar behaviour as our data (e.g. their Fig. 2 and discussion in Ferguson \& Dotter 2008). In particular, they stress that the regime around $\mathrm{C} / \mathrm{O}=1$ is the most sensitive one that should be resolved well.

Table 3 lists the relative abundances of the metals for these various $\mathrm{C} / \mathrm{O}$ ratios, starting with the standard solar (Sets 2-6) or $\alpha$-enhanced (Sets 8-12) composition, as given in Table 2. Note that not only carbon, but also oxygen and nitrogen are enhanced. This is based on the fact that the third dredge-up not only increases carbon, but also these elements to some degree (see Fig. 1 for a typical case). The enhancements are typical values. Heavier elements are assumed to remain unaltered, and therefore the table ends at $\mathrm{Mg}$.

To obtain the appropriate opacity for a point within a stellar model, the following procedure was done: (1) select the set of tables for the calculation, if the base mixture is either solar or $\alpha$ enhanced; (2) interpolate within the $X$-dimension to the present hydrogen abundance for all $Z$ and C/O-values; (3) interpolate to the present $Z$ values; and finally (4) interpolate to the correct $\mathrm{C} / \mathrm{O}$-ratio.

\subsubsection{Approximative molecular opacities}

In addition to the low-temperature opacity tables discussed above, we also had access to tables for carbon-enriched mixtures computed according to Marigo (2002) and kindly provided to us by Marigo. Here, the carbon enhancement was simply added to the base solar composition, beginning at $Z=0.02$. We used them for initial test calculations; some of these will be compared below to our standard calculations in which the tables of Sect. 3.2.1 were employed.

The availability of the approximative and the "ab-initio" tables allows a brief comment on the quality of the approximation. We show the comparison for a very metal-rich mixture of $Z=0.04(X=0.7)$ with an additional amount of carbon of 0.06 (summing up to a total metal fraction of 0.10 ) in Fig. 3. The $\log R$-value is -3 in this plot. The upper panel shows the Marigo and WSU $\log \kappa$, the lower panel shows the relative difference. The agreement is indeed fairly satisfying, even for this extreme carbon enhancement. The global behaviour as a function of temperature is recovered by the approximative opacities, and in the interesting temperature range $3.3 \lesssim \log T \lesssim 3.8$ the deviation remains below a factor 3 at low absolute values. At temperatures above $\log T \gtrsim 3.8$ differences between the computations are not understood, however we use OPAL tables at these temperatures. Overall, we conclude that the Marigo approximation is well suited to describe the basic effects of carbon-enhancement on the temperatures of AGB stars.

\subsection{Mass loss}

Mass loss is a decisive aspect of AGB evolution since it determines how and when the TP-AGB phase ends, what yields can be expected from intermediate-mass stars, and since it also influences possible nuclear reactions at the bottom of the convective envelope. In the absence of a complete theory for mass loss, simple mass loss formulas are implemented in stellar codes. They are obtained by fitting either empirical data or, if available, theoretical mass loss models. The most widely known formulas used for AGB evolution calculations are those by Vassiliadis \& Wood (1993) and Bloecker (1995a). In addition to mass loss on the AGB, including the "superwind" phase, which terminates this evolutionary phase, also the AGB - post-AGB transition phase, the post-AGB evolution at increasingly higher $T_{\text {eff }}$, and the previous phases should be covered, although mass loss there is rather insignificant when compared to that on the AGB.

In this investigation we employed the following mass loss prescriptions: As the basic formula, in particular for the RGB evolution, the Reimers relation (Reimers 1975) is used.

$\dot{M}_{\mathrm{R}}=-4 \times 10^{-13} \frac{\left(L / L_{\odot}\right)\left(R / R_{\odot}\right)}{\left(M / M_{\odot}\right)} \eta_{\mathrm{R}}$

where $\eta_{\mathrm{R}}$ was set to 1.0 for stars with initial mass larger than $1.7 M_{\odot}$, following Bloecker (1995a). For models with smaller ZAMS masses, a more standard value of 0.4 has been chosen as in Karakas (2003). In practice, the mass loss resulting from Eq. (3) is insignificant with the exception of the RGB evolution of low-mass stars. 
Table 3. Logarithmic metal abundances of the opacity tables for varying $\mathrm{C} / \mathrm{O}$ ratios.

\begin{tabular}{cccccccccccc}
\hline \hline & Set $^{a}$ & Set 3 & Set 4 & Set 5 & Set 6 & Set 8 & Set 9 & Set 10 & Set 11 & Set 12 \\
el. & $\mathrm{C} / \mathrm{O}=0.9$ & $\mathrm{C} / \mathrm{O}=1.0$ & $\mathrm{C} / \mathrm{O}=1.1$ & $\mathrm{C} / \mathrm{O}=3.0$ & $\mathrm{C} / \mathrm{O}=20.0$ & $\mathrm{C} / \mathrm{O}=0.9$ & $\mathrm{C} / \mathrm{O}=1.0$ & $\mathrm{C} / \mathrm{O}=1.1$ & $\mathrm{C} / \mathrm{O}=3.0$ & $\mathrm{C} / \mathrm{O}=20.0$ \\
\hline $\mathrm{C}$ & 8.90 & 8.97 & 9.03 & 9.48 & 10.47 & 9.35 & 9.42 & 9.48 & 9.89 & 10.87 \\
$\mathrm{~N}$ & 8.14 & 8.18 & 8.22 & 8.56 & 8.91 & 8.45 & 8.50 & 8.56 & 8.92 & 9.29 \\
$\mathrm{O}$ & 8.95 & 8.97 & 8.99 & 9.00 & 9.17 & 9.40 & 9.42 & 9.44 & 9.42 & 9.57 \\
$\mathrm{Ne}$ & 8.07 & 8.07 & 8.07 & 8.07 & 8.07 & 8.47 & 8.47 & 8.47 & 8.47 & 8.47 \\
$\mathrm{Na}$ & 6.33 & 6.33 & 6.33 & 6.33 & 6.33 & 6.33 & 6.33 & 6.33 & 6.33 & 6.33 \\
$\mathrm{Mg}^{b}$ & 7.58 & 7.58 & 7.58 & 7.58 & 7.58 & 7.98 & 7.98 & 7.98 & 7.98 & 7.98 \\
$\ldots$ & $\ldots$ & $\ldots$ & $\ldots$ & $\ldots$ & $\ldots$ & $\ldots$ & $\ldots$ & $\ldots$ & $\ldots$ & $\ldots$ \\
\hline
\end{tabular}

${ }^{a}$ Sets 1 and 7 are omitted as they correspond to the standard solar-scaled and $\alpha$-enhanced distributions of Table $2 ;^{b}$ table ends at Mg since elements heavier than oxygen remain as in the base mixtures.

Once on the AGB, observed mass loss rates are higher than the standard Reimers wind would indicate. The common picture is that the winds are driven by radiation-dust interactions, where the dust production itself is triggered or enhanced by radial pulsations (see Wallerstein \& Knapp 1998; Sedlmayr $\&$ Winters 1997). At the present time, thorough theoretical radiation-hydrodynamical models including dust production are available only for carbon-rich chemical compositions, in which nearly all oxygen is bound in $\mathrm{CO}$, and the excessive carbon gives rise to carbon-based molecules and dust. The Berlin group has published both models and fitting formulas for such cases (e.g. Fleischer et al. 1992; Arndt et al. 1997; Winters et al. 1997). We are employing here the mass loss rate by Wachter et al. (2002),

$$
\begin{aligned}
\log \dot{M}_{\mathrm{AGB}}= & -4.52+2.47 \cdot \log \left(10^{-4} \frac{L}{L_{\odot}}\right) \\
& -6.81 \cdot \log \left(\frac{T_{\mathrm{eff}}}{2600 K}\right)-1.95 \cdot \log \left(\frac{M}{M_{\odot}}\right) .
\end{aligned}
$$

This formula does not include any dependence on the actual C/O-ratio as did an earlier formulation by Fleischer (1994), which was used by Wagenhuber (1996), the first work to include a mass loss rate based on such models. Wachter et al. (2002) showed that the dependence of the mass loss rate on the $\mathrm{C} / \mathrm{O}$ ratio is weak enough to be ignored in comparison with all other uncertainties. This statement, however, was obtained from investigating models at solar metallicity. In Wachter et al. (2008) the same models were used to derive similar fit formulas for LMC and SMC metallicities. Although the dependence on $\mathrm{C} / \mathrm{O}$ was again neglected, the coefficients in the equations corresponding to Eq. (4) are different. We speculate that these coefficients also contain the hidden effect that for a given $\mathrm{C} / \mathrm{O}$-ratio the absolute number of unbound, therefore available carbon atoms differs between different total metallicities. This is, according to Mattsson et al. (2008), the decisive quantity. Since both papers appeared after our computations were already performed, the metallicity dependence could not be taken into account.

The second obvious dependence on pulsation period has been implicitly included in the $L$-term according to the periodluminosity relation by Groenewegen \& Whitelock (1996).

For the case of oxygen stars, i.e. $\mathrm{C} / \mathrm{O}<1$, we used the empirical fitting formula by van Loon et al. (2005) obtained from dust-enshrouded oxygen-rich AGB stars:

$$
\begin{aligned}
\log \dot{M}_{\mathrm{AGB}}= & -5.65+1.05 \cdot \log \left(10^{-4} \frac{L}{L_{\odot}}\right) \\
& -6.3 \cdot \log \left(\frac{T_{\mathrm{eff}}}{3500 \mathrm{~K}}\right) .
\end{aligned}
$$

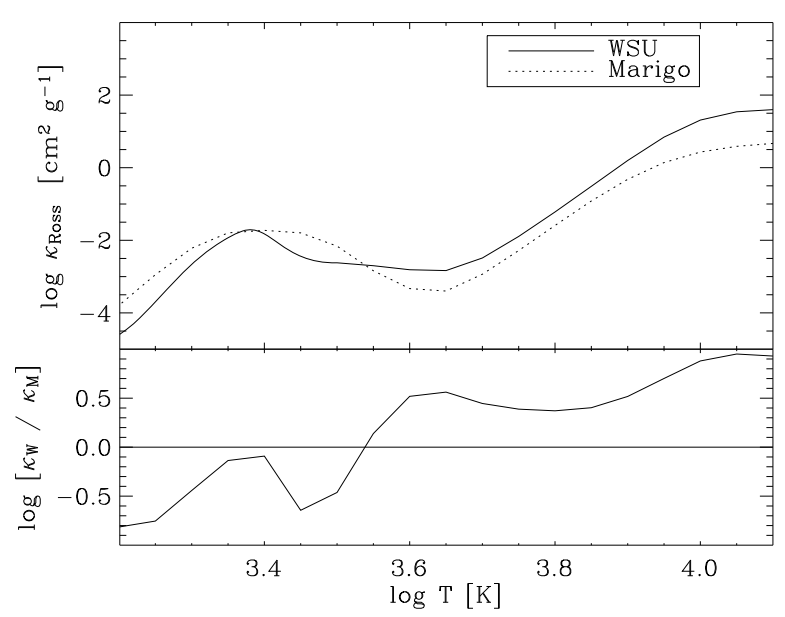

Fig. 3. Comparison of the approximative molecular opacities by Marigo (2002) with those by the WSU group (see Sect. 3.2.1) for a chemical composition with solar-scaled metallicity of $Z=0.04$, enriched by an additional amount of carbon of 0.06 .

This rate is applicable only to stars with a pulsation period $P>400$ days. Similar period cuts of 400 and 100 days have been employed by Vassiliadis \& Wood (1993) and Bloecker (1995a). In accordance with the latter reference, we use the Ostlie \& Cox (1986) estimate of the pulsation period for Mira variables of given mass and radius, and apply Eqs. (4) and (5) if $P>400$ days. For the carbon-rich case, Wachter et al. (2002) actually give a lower critical luminosity, but in our calculations it turned out that models reach the critical pulsation period as oxygen-rich stars and only later become carbon-stars at a higher luminosity.

As a star leaves the AGB after a "superwind" has removed most of its envelope, $T_{\text {eff }}$ increases and the above formulae lead to a quickly vanishing stellar wind. For this transition to the post-AGB phase no suitable mass loss formula is available. Schönberner \& Steffen (2007) argue, based on hydrosimulations of dust envelopes around evolving post-AGB stars, that the strong mass loss should pertain up to effective temperatures of 5000 or $6000 \mathrm{~K}$. We mimic this by keeping the AGBwind mass loss rates until $P$ has decreased to 150 days. From there to $P=100$ days, taken to be the beginning of the postAGB phase, a linear transition to the post-AGB wind is done. From there on, we again use the Reimers relation (Eq. (3)), or, if the rate is larger, the radiation-driven wind formula already used by Bloecker (1995b), and based on Pauldrach et al. (1988), namely:

$\dot{M}_{\mathrm{CSPN}}=-1.29 \times 10^{-15} L^{1.86}$ 


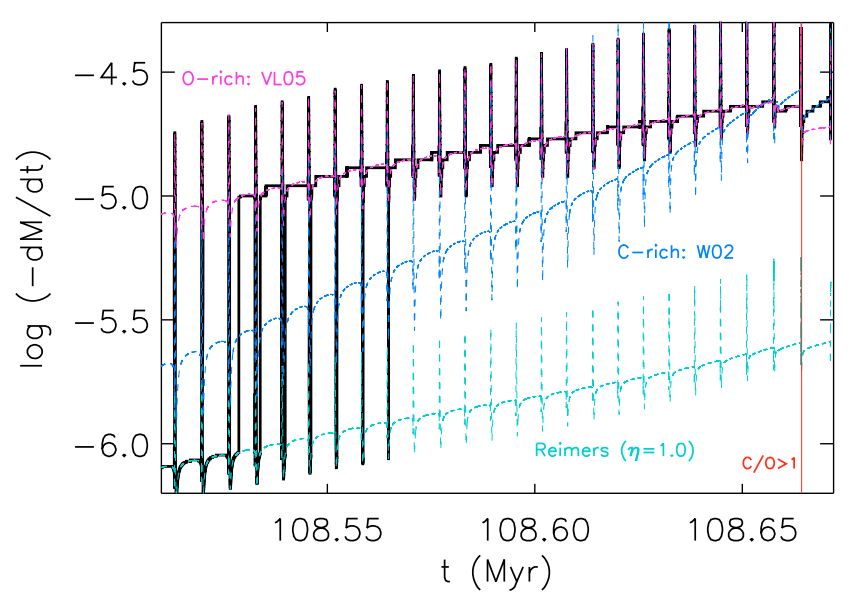

Fig. 4. Logarithmic mass-loss rate with respect to time (in million years) during the TP-AGB evolution of a $5 M_{\odot}$ model with solar type composition $(Z=0.02)$. The actually adopted mass loss value is given by the thick black line. The colored dashed lines give the rates from each of the individual prescriptions according to Eq. (3) (Reimers), 4 (W02), or 5 (VL05). The vertical red line marks the beginning of the carbon-rich phase.

Figure 4 shows an illustrative example about the relative size of the mass loss due to the various prescriptions and the rate actually used in the evolution of a $5 M_{\odot}$ star of solar-like composition on the AGB. On the early AGB a Reimers wind of $\eta_{\mathrm{R}}=1$ is assumed; after three of the thermal pulses shown in this figure, the critical $400 \mathrm{~d}$ pulsation period is reached and mass loss according to Eq. (5) is applied. This leads to an increase in $\dot{M}$ by an order of magnitude. Before the last TP of the figure dredge-up turns the model into a carbon star and we switch to the dust-driven wind of Eq. (4) (W02), which is slightly above the van Loon mass loss rate (VL05). Notice that both types of wind show a drop at this point, due to an increase in effective temperature which is caused by the carbon-enhanced opacities. This is one of the events we encountered, where a value of $\mathrm{C} / \mathrm{O}>1$ does not lead to the expected decrease in $T_{\text {eff }}$, and which will be discussed below. Note also the steeper rise of $\dot{M}$ with time, i.e. luminosity, for the dust-driven wind (W02).

\section{Results of the calculations}

\subsection{Overview about pre-AGB evolution}

Although the evolution up to the TP-AGB phase is of less interest in the context of this paper, we briefly discuss these initial phases. Tables A.1 and A.2 list lifetimes on the main-sequence, the RGB (to be understood as the time between the end of central hydrogen and the beginning of central helium burning), the core helium burning, and until the onset of the first thermal pulse. In addition, total mass and core mass at the first TP are included.

In Fig. A.1 we compare main-sequence lifetimes of our models with those by Karakas (2003, K03) and Vassiliadis \& Wood (1993, VW93) for which chemical compositions agree. Both sets include metallicities of 0.004 and $0.008, \mathrm{~K} 03$ also has a case with $Z=0.02$, but VW93 provides $Z=0.016$ only. The latter set has always $Y=0.25$, while $\mathrm{K} 03$ has varying helium contents of $0.2476,0.2551,0.2928$ for the three metallicities (from low to high $Z$ ). In addition, both sets exclude core overshooting, and have older opacities and nuclear reaction rates. At the lowest metallicity, where composition differences are smallest, main-sequence ages between all three sets agree to better than $10 \%$. The generally longer lifetimes of our models with respect to $\mathrm{K} 03$, most pronounced for $Z=0.02$, can be understood as a result of overshooting. Indeed, when repeating the calculations without overshooting (black triangles in the lowest left panel of Fig. A.1) both sets agree to a few per cent. The generally longer lifetimes of VW93 models can be traced back to their higher hydrogen abundance, which not only provides more nuclear fuel, but also decreases surface temperatures due to higher opacities and, because of the lowered molecular weight, lowers the luminosities of the models.

For the core helium burning lifetimes the discrepancies are much larger. Our models for stars above $2 M_{\odot}$ have lifetimes shorter by several factors of $10 \%$. Similar discrepancies exist between the K03 and VW93 models. Our lifetimes agree best with those of the Padova library (Girardi et al. 2000), which we also consulted for comparison. As already discussed by $\mathrm{K} 03$, this phase is very uncertain due to its dependence on the ${ }^{12} \mathrm{C}(\alpha, \gamma){ }^{16} \mathrm{O}$ reaction rate and the treatment of overshooting and semi-convection.

The ignition of helium could be followed in all our models, independent of whether this happened under non-degenerate conditions in the intermediate-mass stars, or as a core helium flash in low-mass stars. No artificial setup of post-flash models was therefore needed (Serenelli \& Weiss 2005).

The pre-AGB phase ends with the first thermal pulse, defined as the first pulse in which a helium luminosity of $10^{4} L_{\odot}$ is reached. This definition coincides either with the first appearance of the pulse-driven convection zone, or with one pulse earlier than that. In general, such a pulse is not yet fully developed. At this stage, the mass of the hydrogen-free core is a quantity of interest, as the future growth of the core is important for the initial-final-mass relation. In Fig. A.1, right column, we compare the core mass at the first thermal pulse with the values by $\mathrm{K} 03$. While they agree very well for the intermediate mass stars, ours are systematically lower for low-mass stars. In particular they show the characteristic minimum value around $2 M_{\odot}$, while the K03 models show only a very shallow variation below $2.5 M_{\odot}$. In the case of $Z=0.02$ we also added values obtained by Miller Bertolami (2008, private communication). They are shown as blue diamonds and agree very well with our numbers. Miller Bertolami uses the same overshooting prescription as we do. Ignoring overshooting, core masses tend to be smaller for those stars with convective cores on the main sequence. These core masses are displayed for the same metallicity as black triangles. In view of the fact that K03 did not include overshooting, the agreement between her core masses and those of our models is in fact another sign of discrepancy.

We close this overview by mentioning that the influence of $\alpha$-enhancement at identical $Z$ is such that lifetimes are generally shorter and core masses at the first TP slightly larger (see Tables A.1 and A.2).

\subsection{AGB synopsis}

\subsubsection{Overall properties}

The evolution of a sample model $\left(M=2 M_{\odot}, Z=0.02\right.$ with solar metal ratios) along the AGB is shown in Fig. 5. We plot key quantities such as luminosity, effective temperature, pulsation period, mass loss rate, and C/O-ratio at the surface. The model experiences in total 15 TPs; the third dredge-up (3du) starts at the second TP and continues until the end of the AGB; the mass loss rate switches from an enhanced Reimers wind to 

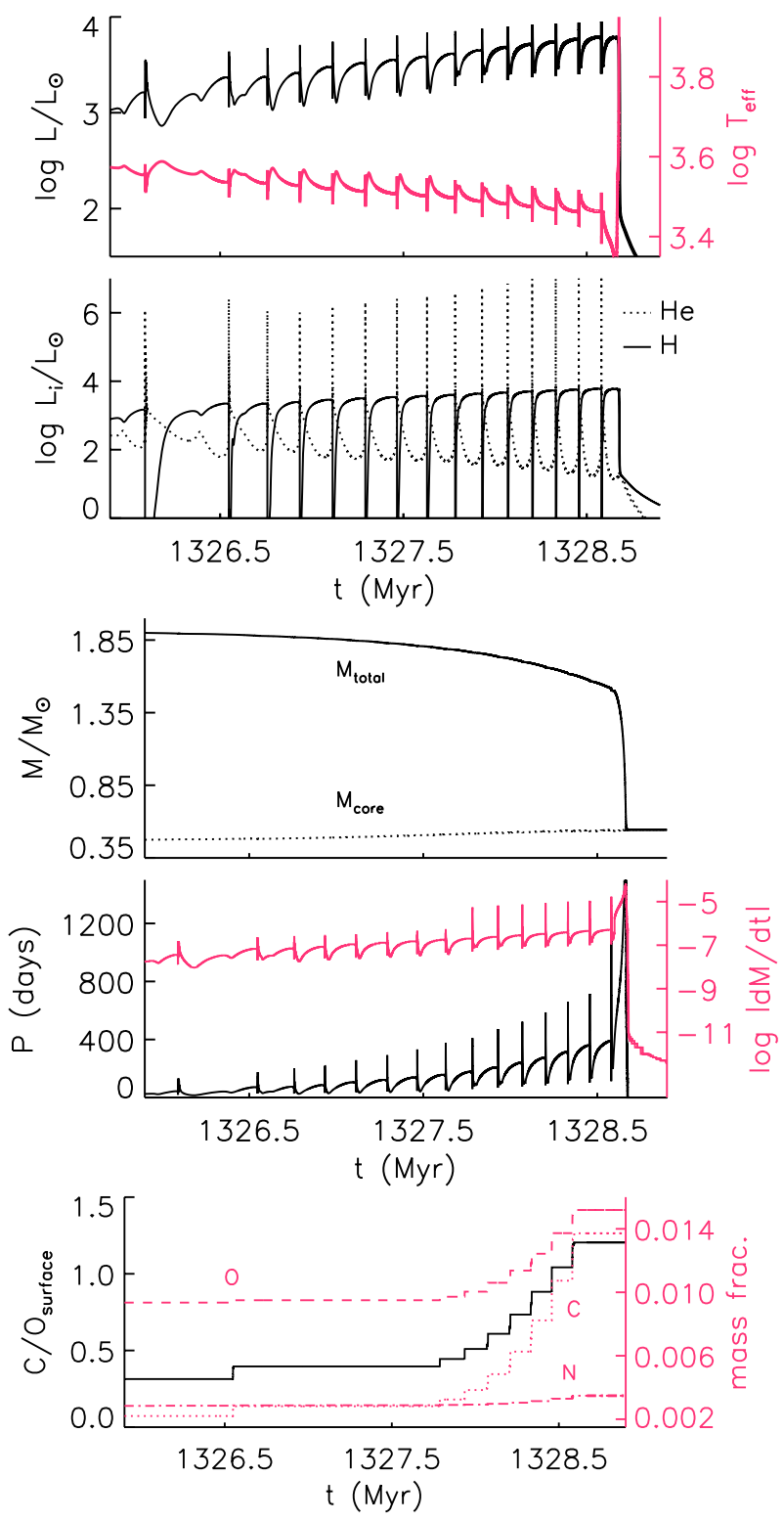

Fig. 5. Various quantities of a model with initial mass $M=2 M_{\odot}, Z=$ 0.02 (solar metal ratios) from the beginning of the AGB until the early post-AGB phase. From top to bottom the panels show $\log L$ and $\log T_{\text {eff }}$, $\log L_{\mathrm{H}}$ (solid) and $\log L_{\mathrm{He}}$ (dotted), total and core mass, pulsation period and $\log \dot{M}, \mathrm{C} / \mathrm{O}-$ ratio and abundances of $\mathrm{C}, \mathrm{N}$, and $\mathrm{O}$.

$\dot{M}_{\mathrm{AGB}}$ according to Eq. (5) at TP 9, when the pulsation period reaches the critical 400 days, and finally to the dust-driven $\dot{M}_{\mathrm{AGB}}$ (Eq. (4)) stage once $\mathrm{C} / \mathrm{O}>1$ is reached during the second last TP. At this epoch a clear drop in $T_{\text {eff }}$ is visible, which is the direct consequence of the carbon-rich opacity tables we are using. The strong dust-driven wind leads to a "superwind" which removes within one interpulse period more than $1 M_{\odot}$. The remaining, nearly bare core starts the post-AGB evolution at much higher $T_{\text {eff }}$ and a reduced stellar wind. This model does not experience $\mathrm{HBB}$, as the temperature at the bottom of the convective envelope reaches temperatures of only around $30 \mathrm{MK}$, much less than the typical HBB temperature of $50 \mathrm{MK}$.

For this metallicity of $Z=0.02$ Table 4 summarizes some key properties of our models along the AGB. Equivalent tables for the other four metallicities are contained in the on-line material.

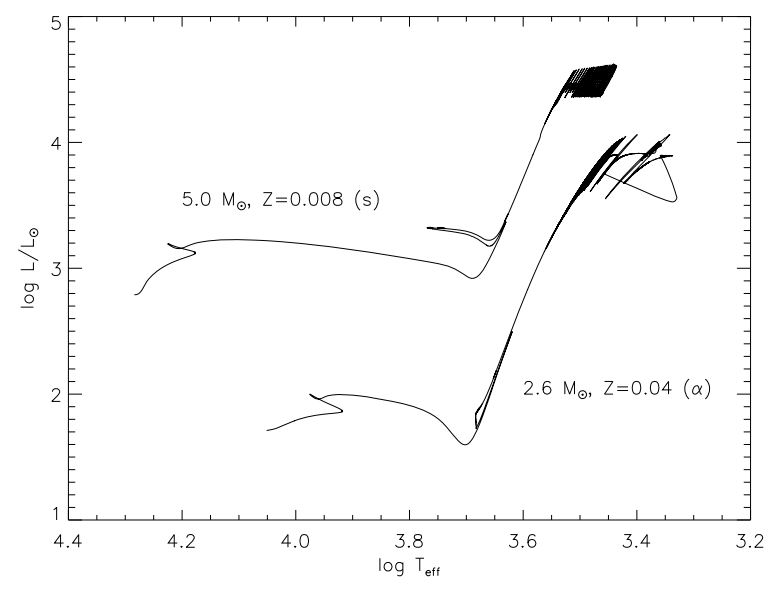

Fig. 6. Evolution of two of our models. Shown are stars of $5 M_{\odot}$ with $Z=0.008$ (solar-scaled) and 2.6 $M_{\odot}$ with $Z=0.04$ ( $\alpha$-enhanced).

Despite all numerical improvements, we still encountered convergence problems such that only part of the models could be evolved to the very end of the AGB, which we defined as the point when the model leaves the AGB towards hotter $T_{\text {eff }}$ and the pulsation period has dropped below 100 days. These convergence problems can be traced back to a dominance of radiation pressure within the convective envelopes, and have been reported and identified as early as in Wood \& Faulkner (1986), but also by, e.g., Karakas (2003), Herwig (2005, private communication), Miller Bertolami \& Althaus (2006b), and Karakas \& Lattanzio (2007). In some cases the convergence problems could be circumvented by artificially stripping off the remaining envelope and relaxation of the resulting model, by increasing the mixing length parameter, or by shifting more mass into an energetically inert outer envelope (which our models do not have). None of these methods worked satisfactorily for us, such that we decided to stop the calculation when convergence problems became insurmountable. In most cases, in particular for lower masses, the models evolved to virtually the end of the AGB phase. For example, the $1 M_{\odot}$ star of Table 4 crashed during the final flash, when it was departing from the AGB during the third dredge-up. In such cases, due to the amount of mass loss and the size of the remaining envelope, an estimate of the TPs and time still needed to complete the TP-AGB phase can be safely done. This estimate is given in Table 4 as the second last column ( $t_{\mathrm{e}}$; missing TPs in brackets) and added in the final column to the actual AGB lifetime at which the end of the computations was reached $\left(t_{\mathrm{mod}}\right)$. We follow the approach by Karakas (2003). From Tables 4 and B.1-B.4 it is evident, that up to $M=4 M_{\odot}$ at most 2 TPs are missing for the complete AGB evolution, but that for higher masses a substantial part of the AGB is missed. Altogether 100 out of 110 sequences reached that advanced stage, with 79 missing less than 3 TPs. For the two lowest metallicities some models experienced convergence problems so early on the TP-AGB that we did not include them in these tables.

We show as examples the evolutionary paths of two of our models in the Hertzsprung-Russell-Diagram (Fig. 6), calculated with all physical details discussed in Sect. 3. The $5 M_{\odot}$ star (of the typical LMC composition V) experiences 38 TPs in total. Dredge-up occurs from the first TP, and is sufficient to turn the star into a carbon star. The dredge-up is competing, however, with strong HBB between TPs 9 and 24, which reduces $\mathrm{C} / \mathrm{O}$ to a final value close to 1 . During the last three pulses the 
Table 4. Basic model properties along the AGB for a total metallicity of $Z=0.02$ and solar and $\alpha$-enhanced metal distributions.

\begin{tabular}{|c|c|c|c|c|c|c|c|c|c|c|c|c|}
\hline \multirow[t]{2}{*}{$M_{\mathrm{ZAMS}}^{a}$} & \multirow{2}{*}{$\begin{array}{c}\text { No } \\
\text { TPs }^{b}\end{array}$} & \multirow[t]{2}{*}{$M_{\mathrm{c}}(1)^{c}$} & \multirow[t]{2}{*}{$M_{\mathrm{c}}(3 \mathrm{du})^{d}$} & \multirow{3}{*}{$\begin{array}{c}\text { No } \\
\mathrm{TP}_{3 \mathrm{du}}(\mathrm{i})^{e}\end{array}$} & \multirow[t]{2}{*}{$\mathrm{C} / \mathrm{O}_{\mathrm{f}}^{f}$} & \multirow[t]{2}{*}{$M(\mathrm{f})^{g}$} & \multirow[t]{2}{*}{$M_{\mathrm{c}}(\mathrm{f})^{h}$} & \multirow{2}{*}{$\begin{array}{c}\text { No } \\
\operatorname{TP}_{\mathrm{HBB}}(\mathrm{i})^{i}\end{array}$} & \multirow{2}{*}{$\begin{array}{c}\text { No } \\
\mathrm{TP}_{\mathrm{HBB}}(\mathrm{f})^{k}\end{array}$} & \multicolumn{3}{|c|}{ TP-AGB lifetime $\left(10^{3} \mathrm{yrs}\right)^{l}$} \\
\hline & & & & & & & & & & $t_{\bmod }$ & $\approx t_{\mathrm{e}}(\mathrm{TPs})$ & $t_{\mathrm{TP}}$ \\
\hline \multicolumn{12}{|c|}{$Z=0.02 /$ solar (mixture III) } & \\
\hline 1.0 & 2 & 0.501 & 0.508 & 1 & 0.547 & 0.547 & 0.508 & - & - & 327.675 & $36(0)$ & 364 \\
\hline 1.2 & 5 & 0.509 & - & - & 0.361 & 0.608 & 0.524 & - & - & 537.625 & $25(0)$ & 563 \\
\hline 1.5 & 8 & 0.511 & 0.526 & 5 & 0.724 & 0.637 & 0.539 & - & - & 911.274 & $15(0)$ & 926 \\
\hline 1.6 & 9 & 0.508 & 0.522 & 5 & 0.753 & 0.888 & 0.538 & - & - & 1066.336 & $69(0)$ & 1135 \\
\hline 1.8 & 8 & 0.496 & 0.503 & 2 & 1.252 & 1.031 & 0.524 & _ & - & 1372.882 & $159(1)$ & 1532 \\
\hline 2.0 & 15 & 0.478 & 0.484 & 2 & 1.204 & 0.543 & 0.543 & _- & - & 2581.483 & - & 2581 \\
\hline 2.6 & 14 & 0.518 & 0.533 & 5 & 1.426 & 1.802 & 0.560 & - & - & 1560.967 & $311(2)$ & 1872 \\
\hline 3.0 & 12 & 0.596 & 0.596 & 1 & 1.407 & 2.029 & 0.617 & - & - & 693.755 & $141(2)$ & 835 \\
\hline 4.0 & 14 & 0.765 & 0.766 & 2 & 0.711 & 2.137 & 0.783 & - & - & 156.353 & $54(3)$ & 210 \\
\hline 5.0 & 29 & 0.830 & 0.831 & 2 & 1.051 & 2.354 & 0.850 & - & - & 169.289 & $60(7)$ & 229 \\
\hline 6.0 & 34 & 0.928 & 0.928 & 1 & 0.226 & 3.178 & 0.937 & 3 & 29 & 98.985 & $71(23)$ & 170 \\
\hline \multicolumn{13}{|c|}{$Z=0.02 / \alpha$-enhanced (mixture IV) } \\
\hline 1.0 & 2 & 0.502 & - & - & 0.164 & 0.551 & 0.505 & - & - & 319.945 & $43(0)$ & 363 \\
\hline 1.2 & 5 & 0.508 & 0.518 & 4 & 0.384 & 0.561 & 0.521 & - & - & 529.722 & $12(0)$ & 542 \\
\hline 1.5 & 8 & 0.514 & 0.530 & 5 & 1.834 & 0.542 & 0.540 & - & - & 929.208 & - & 929 \\
\hline 1.6 & 9 & 0.513 & 0.523 & 4 & 0.859 & 0.545 & 0.544 & - & - & 1018.272 & - & 1018 \\
\hline 1.8 & 11 & 0.501 & 0.509 & 4 & 1.240 & 0.551 & 0.540 & - & - & 1412.476 & $2(0)$ & 1414 \\
\hline 2.0 & 14 & 0.488 & 0.494 & 2 & 1.248 & 0.629 & 0.537 & - & - & 2196.938 & $9(0)$ & 2206 \\
\hline 2.6 & 15 & 0.513 & 0.527 & 5 & 1.453 & 1.853 & 0.547 & - & - & 1821.007 & $412(3)$ & 2233 \\
\hline 3.0 & 14 & 0.585 & 0.588 & 2 & 1.332 & 1.922 & 0.612 & - & - & 907.181 & 207 (2) & 1114 \\
\hline 4.0 & 13 & 0.766 & 0.766 & 1 & 0.681 & 1.958 & 0.774 & - & - & 158.834 & 37 (2) & 196 \\
\hline 5.0 & 21 & 0.846 & 0.846 & 2 & 0.541 & 3.215 & 0.860 & - & - & 117.461 & 94 (15) & 211 \\
\hline 6.0 & 37 & 0.946 & 0.946 & 1 & 0.252 & 3.260 & 0.954 & 1 & 32 & 91.610 & $58(19)$ & 150 \\
\hline
\end{tabular}

${ }^{a}$ Initial mass on ZAMS; ${ }^{b}$ number of thermal pulses; ${ }^{c}$ core mass at first TP; ${ }^{d}$ mass at onset of third dredge-up; ${ }^{e}$ TP number of $3 \mathrm{du}$ onset; ${ }^{f}$ final $\mathrm{C} / \mathrm{O}$ ratio; ${ }^{g}$ final mass; ${ }^{h}$ final core mass; ${ }^{i}$ first TP with $\mathrm{HBB} ;{ }^{k}$ last TP with HBB; ${ }^{l}$ TP-AGB lifetimes (see text). Equivalent tables for all other mixtures are contained in the on-line material (Tables B.1-B.4).

convergence problems increased and the evolution in the HRD became irregular. We do not show these last TPs.

The second star with $M=2.6 M_{\odot}$ and an $\alpha$-enhanced mixture of $Z=0.04$ (possibly representative for an extreme bulge composition) experienced 16 TPs and dredge-up after the 9th TP, which increased $Z$ to 0.048 . The decrease of $T_{\text {eff }}$ between pulses, clearly visible in Fig. 6 is the consequence of this overall metallicity increase due to dredge-up of mainly $\mathrm{C}$ and $\mathrm{O}$, and to a lesser extent of $\mathrm{N}$. Although $\mathrm{C} / \mathrm{O}$ more than doubles to 0.432 , the star does not become a carbon star; mass loss is strong according to Eq. (5). This star finally leaves the AGB during the last TP, and turns to hotter temperatures, but the calculations ended before it reached the post-AGB phase, due to the mentioned convergence problems.

\subsubsection{Influence of composition}

Our models recover some previously known trends with mass and metallicity. The $3 \mathrm{du}$ is more pronounced for lower metallicity and higher mass. This is also reflected in the $\mathrm{C} / \mathrm{O}$-values. For the highest masses, HBB reduces carbon. It sets in earlier for lower metallicity. While this is consistent with, e.g., Karakas (2003), the C/O ratios reached in her models are generally higher than ours, up to 20 compared to about 5 in our case. It should be kept in mind that the amount of dredge-up depends crucially on the method to obtain it: while we use overshooting, Karakas (2003) achieves it by extending the convective regions to a point of marginal stability, but does so only for the lower boundary of the convective envelope. Her models do not contain any overshooting from the pulse-driven convective layers, and therefore less $\mathrm{C}$ and $\mathrm{O}$ are mixed from the core into the intershell layers. As Herwig (2000, Figs. 11 and 12) has demonstrated, overshooting leads to relatively more oxygen than carbon enhancement, and therefore to lower $\mathrm{C} / \mathrm{O}$-ratios. This is a reason for our lower surface $\mathrm{C} / \mathrm{O}$-ratios. According to Herwig (2000) and Werner \& Herwig (2006) the present surfaces of hot, hydrogen-deficient post-AGB stars of type PG1159 and [WC] have formerly been interior layers of AGB stars, uncovered by late AGB or post-AGB thermal pulses. If that is indeed the case, these stars present a valuable test for the intershell composition of stars along the AGB. In most cases (see Table 1 of Werner \& Herwig 2006), the observed C/O-ratio is below 10, and clusters around values of 3-5, which would confirm the lower values resulting from the inclusion of overshooting from the He-driven convective zone. Similarly, C/O-ratios in planetary nebulae are, in spite of all difficulties in determining them, in most cases well below our maximum value of 5 (see Liu et al. 2004, for a comprehensive and thorough analysis), and never reach values close to 10 (see also Péquignot et al. 2000, for two PNe in the Sagittarius dwarf galaxy).

HBB occurs in the models of Karakas (2003) and in those by Herwig (2004a) around $M=3.5 M_{\odot}$; our models show this only at higher masses $\left(M=5 M_{\odot}\right.$ and above).

A new feature of our grid is the use of solar-scaled and $\alpha$-enhanced metal distributions. We find that there is no significant influence on the number of TPs or the occurrence of $3 \mathrm{du}$ and HBB. The core mass at the onset of TPs and the 3 du tends to be larger by a few $10^{-3} M_{\odot}$ and the final C/O ratio tends to be lower. Since C/O is initially only 0.19 in the $\alpha$-enhanced mixtures, it is clear that an enhanced $3 \mathrm{du}$ is needed to convert the model into a carbon star. As a general rule, the $\alpha$-enhanced models need about 1-2 TPs more to achieve a similar C/O-ratio. This is the reason why the $2.6 M_{\odot}$ model of Fig. 6 does not turn into a carbon star in spite of dredge-up. 


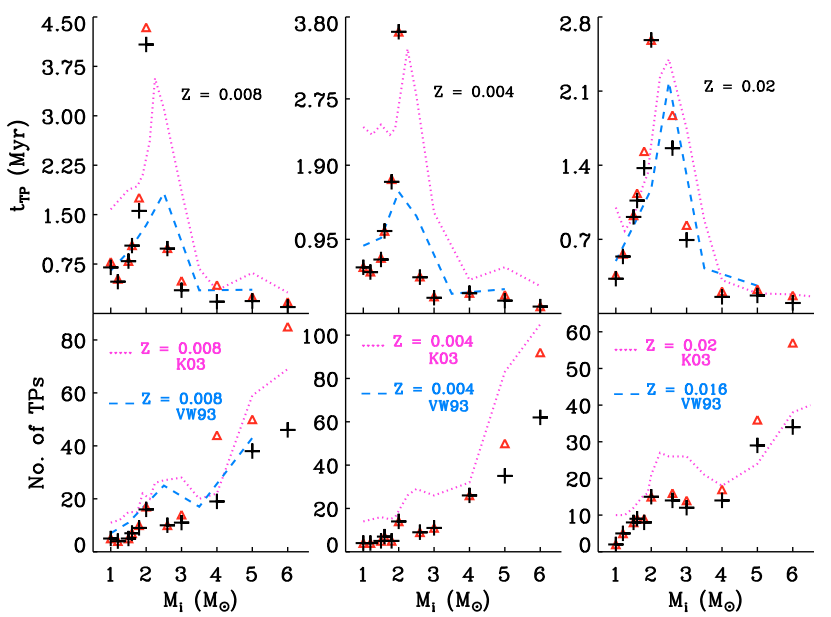

Fig. 7. Top panels: lifetimes on the TP-AGB for our models (black crosses) in comparison with those by K03 (red dotted lines) and VW93 (blue dashed lines) for the three metallicities in common. The most metal-rich mixture of VW93 is $Z=0.016$ instead of 0.02 , however. The red triangles are our estimates for the total lifetimes $t_{\mathrm{TP}}$ of our models taking into account $t_{\mathrm{e}}$ of Table 4. Bottom panels: comparison of the number of thermal pulses. Symbols and lines as above. Data for the two more metal-rich mixtures of VW93 are not available.

\subsubsection{Comparison with other models}

The comparison with the results of Karakas (2003) and Vassiliadis \& Wood (1993) with respect to TP-AGB lifetimes and number of thermal pulses is shown in Fig. 7. Our models show the same global behaviour with a maximum of $t_{\mathrm{TP}}$ at the lowest initial mass that starts core helium burning under nondegenerate conditions and therefore has the lowest core mass, which leads to the longest interpulse times. Note, however, that the K03 models for $Z=0.004$ show an untypical behaviour at the lowest $M_{\mathrm{i}}$. Lifetimes and number of thermal pulses of our models are typically lower than in the other two calculations, in particular for higher masses. This is due to our mass loss formulas, which lead to globally higher mass loss rates, in particular after C/O-ratio exceeds unity (Fig. 4). Although the differences in the physical input for the calculations are larger in comparison with VW93, Fig. 7 shows a better agreement with these older calculations. The larger differences between K03 and VW93 (both use the same mass loss description) have no explanation. The influence of the metal distributions (solar-scaled or $\alpha$-enhanced) is shown in Fig. 8. Generally, lifetimes on the TP-AGB are shorter for the $\alpha$-enhanced mixtures, although the number of pulses tends to be slightly higher (see above).

\subsubsection{Final core mass}

As another global quantity we present in this section the final core mass, shown in Fig. 9, at the end of the TP-AGB evolution for all ten mixtures as well as the comparison with the results by $\mathrm{K} 03$ for the three mixtures in common. The comparison shows a generally good agreement with K03 for higher stellar masses, but much lower $M_{\text {core }}$ values at lower stellar masses. This is the consequence of the lower core mass at the first TP, visible also in Fig. A.1, and the effect of our overshooting prescription, which prevents the core from growing substantially. This indicates that our overshooting prescription, in particular always using the same overshooting parameter at all convective boundaries may lead to an overestimate of the effect of overshooting. Salaris et al. (2009) and Lugaro et al. (2003) have argued that

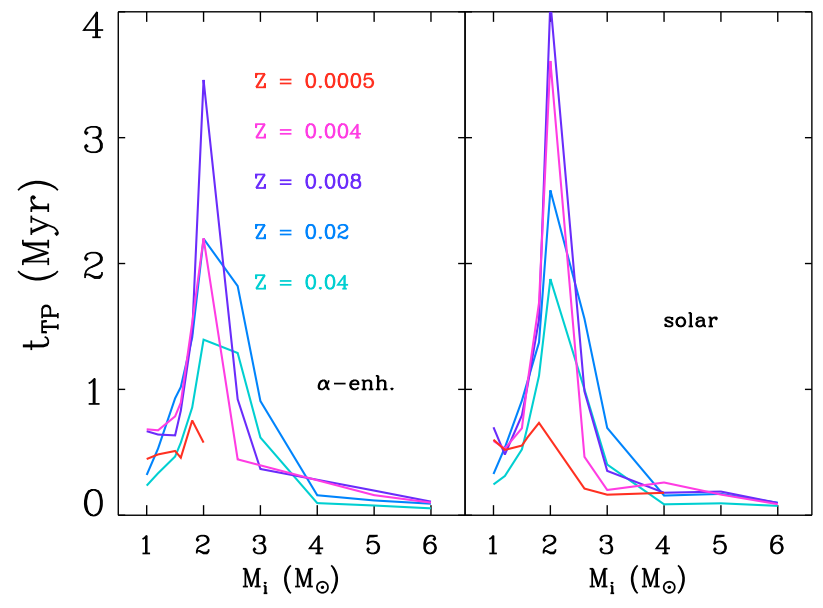

Fig. 8. Lifetimes on the thermally pulsing AGB as function of initial mass for all our models and chemical compositions. Left: $\alpha$-enhanced metal ratios; right: solar-scaled.

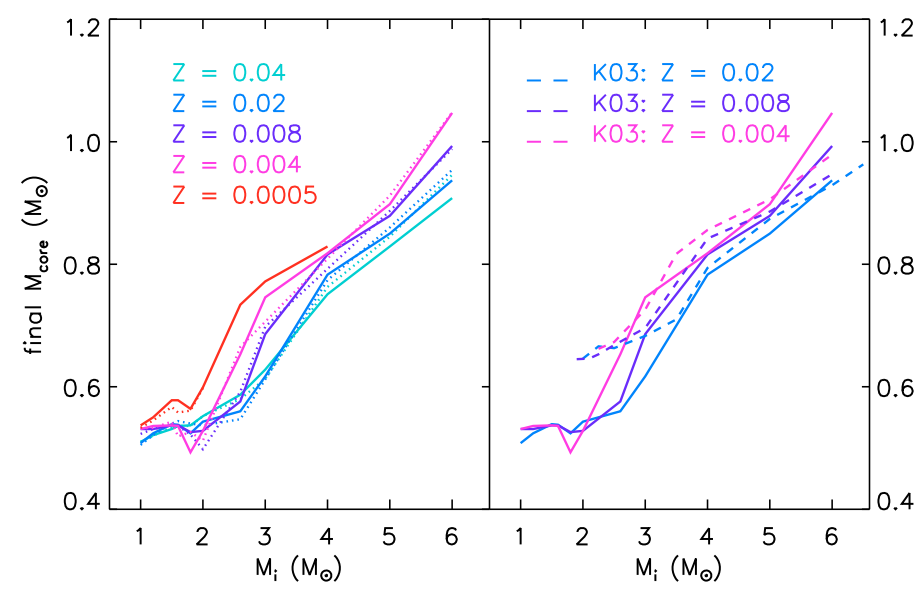

Fig. 9. Left: final core mass of all our models (solid lines: solar-scaled metal ratios; dotted lines: $\alpha$-enhanced). Right: comparison with K03 results (dashed).

the overshooting from the base of the pulse-driven convective layer in the He-shell should be somewhat smaller during the core hydrogen burning to allow the core to grow and to be in better agreement with 3 d-hydrodynamical simulations by Herwig et al. (2007). In terms of the parameter $f$ in Eq. (2) the numerical value should be $f \lesssim 0.01$ instead of our standard value of 0.016 . Indeed, core masses of our models grow during the TP-AGB phase by less than $0.02 M_{\odot}$ except in the mass range between 2 and $4 M_{\odot}$, where the growth reaches $0.04 \cdots 0.06 M_{\odot}$ (more for larger metallicities).

Our predicted initial-final-mass-relation (IFMR) is therefore very close to the relation between initial mass and core mass at the first TP. This is evident from Fig. 10, which shows that our IFMR is a lower envelope - at least at the low-mass end - to the empirical data for clusters with $[\mathrm{Fe} / \mathrm{H}] \approx 0.0$. The initial solar metallicity lies somewhere between the two cases shown. This constitutes a mild discrepancy with the observations for the lower initial mass range, where our predicted IFMR drops below the semi-empirical relation by Weidemann (2000). It agrees well with one by Miller Bertolami (2007; private communication), shown in Salaris et al. (2009), who used a very similar overshooting description as we do. The final core masses of K03, shown in Fig. 9, on the other hand, are higher than the empirical relation for $M \leq 3 M_{\odot}$. 


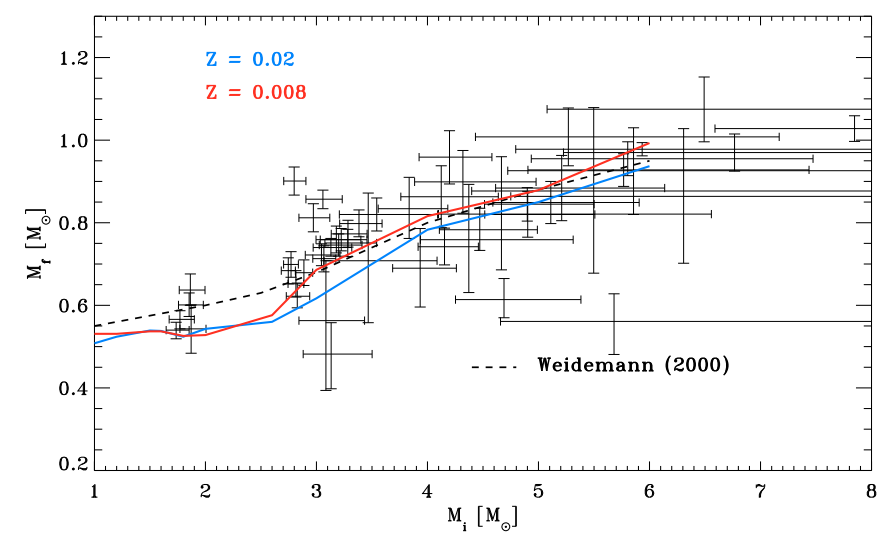

Fig. 10. Our predicted initial-final-mass-relation (solid lines) for $Z=$ 0.02 and 0.008 (solar metal ratios) in comparison with observed open cluster objects and the empirical fit by Weidemann (2000, dashed line). The initial $\left(M_{\mathrm{i}}\right)$ and final $\left(M_{\mathrm{f}}\right)$ mass values and associated error bars are taken from Salaris et al. (2009).

\subsubsection{Analytical fits}

Synthetic AGB calculations make use of analytical relationships between key quantities of full AGB models. Among them, the core-mass - luminosity and the core-mass - interpulse-period relations are two crucial ones. A number of such relations are available in the literature. We mention here the early linear one by Paczyński (1970), the one used by Marigo et al. (1996, M96), which includes a dependency on the envelope composition, and the two most recent and complex relations by Wagenhuber \& Groenewegen (1998, WG98) and Izzard et al. (2004, I04). They are based on the models by Wagenhuber (1996) and Karakas (2003), respectively, and also include dependencies on composition, mixing length parameter, hot bottom burning and dredgeup. Since the latter two effects differ between calculations, it cannot be expected that such detailed relations agree very well with our new models, which incorporate the additional effects of overshooting, opacities for varying C/O-ratios, and mass loss prescription. Nevertheless, a detailed comparison was done by Kitsikis (2008), which we will not repeat here. He found that with respect to the core-mass - luminosity relation, the one by M96 agrees best for low-mass models, because our models have generally lower core masses for a given luminosity. This is related to the low-mass discrepancy found above for the IFMR. For $M_{\text {core }} \gtrsim 0.7 M_{\odot}$ both the I04 and WG98 relations describe our models equally well. The deviations, however, always remain within the 10 to $20 \%$ range. With respect to the core-mass interpulse-period relation the WG98 description fits our models best. Part of the deviation were traced back by Kitsikis (2008) to the effect of the new opacities on luminosity and interpulse time. A new adaption of the WG98 analytical description to our new models seems promising, but awaits completion. If synthetic models require an average accuracy at the $10 \%$ level, the fits by WG98 and I04 might still be used.

\subsection{Effect of varying C/O-ratio}

Since we emphasized a consistent treatment of the carbon enrichment of the envelopes in this work, we will now discuss in detail how this influences the models. We start with a comparison of cases with different sets of opacity tables. This illustrative test was done for a solar composition (mixture III) and a mass of $2 M_{\odot}$. Mass loss was strongly reduced to prevent an amplification of differences in the models due to sensitive mass loss rates. The C/O-ratio and the effective temperature evolution are shown

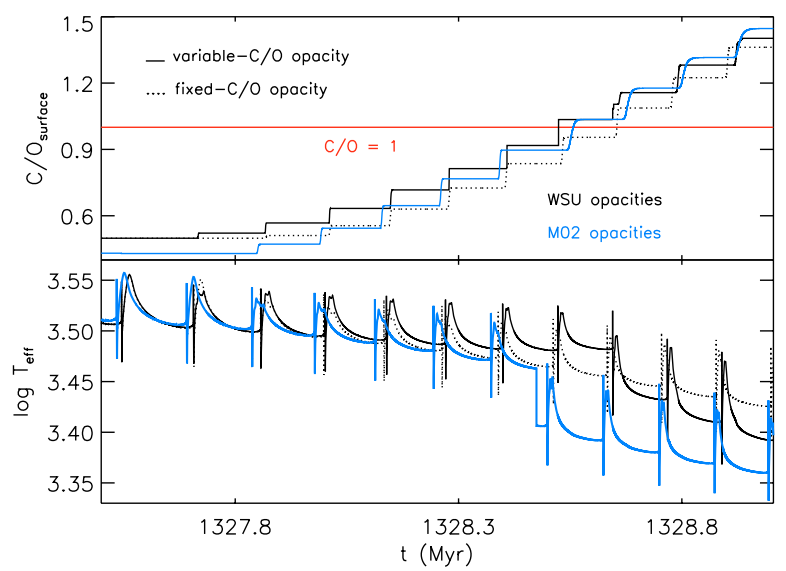

Fig. 11. Evolution of a model with $M=2 M_{\odot}$ and $Z=0.02$ (solar) on the AGB using different sets of opacity tables. Black lines correspond to the new WSU molecular opacities (Sect. 3.2.1), the blue one to the approximative molecular opacities by Marigo (2002, Sect. 3.2.2). The black dotted line refers to calculations where only the total metallicity, but not the change in $\mathrm{C} / \mathrm{O}$ due to the third dredge-up is accounted for.

in Fig. 11 for three different cases. The early AGB evolution up to the 7th TP is not shown. Different 3du histories explain why the $\mathrm{C} / \mathrm{O}$ ratio is not the same in all cases.

The black dotted line corresponds to the use of opacity tables with no specific treatment of the C/O-ratio. This implies that any increase in carbon abundance is taken into account only by using opacity tables of higher metallicity, but still solar metal ratios. The solid blue line is using the Marigo (2002) molecular opacities. Here, any increase in metallicity is ascribed to be due to $\mathrm{C}$ only, irrespective of the actual $\mathrm{C} / \mathrm{O}$ ratio. Generally, the $\mathrm{C} / \mathrm{O}$ ratio is overestimated in these tables, such that the influence on the opacities is exaggerated. These molecular opacities were taken into account in our calculations, when the $\mathrm{C} / \mathrm{O}$ ratio in the models exceeded unity, or when the total metallicity reached $Z \approx 0.03$, which is the case shown in the figure. This implies a sudden jump in opacity, and a corresponding sudden decrease in $T_{\text {eff }}$, visible at $t=1328.5 \mathrm{Myr}$. As such a jump creates in some cases numerical problems in the calculations, we applied the switch to the molecular opacities during the interpulse phase. The lower $T_{\text {eff }}$ then leads to stronger dredge-up in the following TPs. Compared to the equivalent case of ignoring carbon enhancement in the opacities, $T_{\text {eff }}$ drops by up to 0.07 dex during the final 5 TPs. The level of carbon enhancement (upper panel of Fig. 11) is increased with respect to the fixed C/O case.

The WSU opacity tables, introduced in Sect. 3.2.1, in contrast follow the $\mathrm{C} / \mathrm{O}$-ratio in detail, therefore the slow increase in carbon has a smooth influence on $T_{\text {eff }}$ (black solid line). While the WSU molecular opacities also lead to a decrease in $T_{\text {eff }}$, interestingly initially, when $\mathrm{C} / \mathrm{O}$ is approaching unity, this decrease of $T_{\text {eff }}$ is less pronounced than in the case where carbon enhancement is taken into account as a general increase of an otherwise solar-scaled metallicity (dotted black line). However, when looking at Fig. 2, it is evident that indeed up to $\log T_{\text {eff }} \approx$ 3.5 the Rosseland mean opacity first decreases with increasing $\mathrm{C} / \mathrm{O}$ and only for $\mathrm{C} / \mathrm{O}>1$ begins to increase again. Although the physical conditions $(Z, \log R)$ of that figure are not quite the one encountered in the models shown in Fig. 11, this case is very representative. The reason for the initial drop is the reduced number of $\mathrm{TiO}$ and $\mathrm{H}_{2} \mathrm{O}$ molecules, which for O-rich mixtures are a major source of opacity. Eventually, when $\mathrm{C} / \mathrm{O} \gtrsim 1.2$, the $\mathrm{C} / \mathrm{O}$ variable opacities are higher and $T_{\text {eff }}$ drops stronger. In our set of 


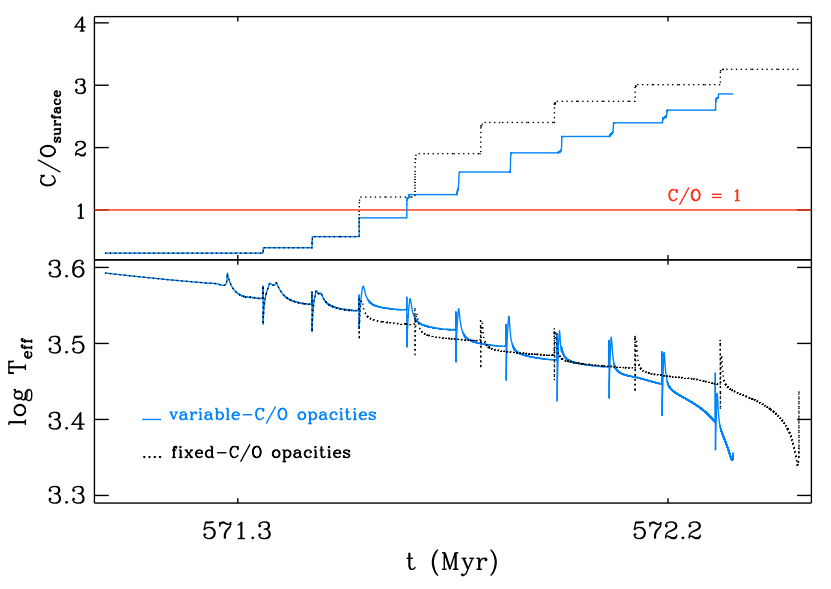

Fig. 12. Evolution of a model with $M=2.6 M_{\odot}$ and $Z=0.008$ (solar) on the AGB using the new WSU molecular opacities. Variable C/Oratios are taken into account in the case indicated by the blue line, and ignored in that shown in black.

tables of Marigo's molecular opacities, we do not have mixtures with $\mathrm{C} / \mathrm{O} \approx 1$, such that the opacity minimum cannot be recovered. This explains why the solid blue line does not show this weaker decrease of the effective temperature as a consequence of carbon enhancement. We emphasize that $T_{\text {eff }}$ in all cases continues to decrease during the TP-AGB phase, but at different rates depending on the detailed treatment of C-enhancement.

A similar case is shown in Fig. 12. Here, we employ our standard mass loss treatment. We compare only the case of ignoring or including variable C/O-ratios. The opacity tables (WSU molecular opacities) are the same in both cases. When dredgeup begins (at the third full TP), the $\mathrm{C} / \mathrm{O}$-variable opacities again lead to higher $T_{\text {eff }}$ as compared to the fixed-C/O opacity tables. As a consequence, dredge-up is more effective for the case, in which the increase in carbon is only taken into account by using opacities for higher $Z$, but still solar $\mathrm{C} / \mathrm{O}$. Only when $\mathrm{C} / \mathrm{O} \gtrsim 2$ (TP no. 6), the increase in carbon is reflected by higher opacities, lower $T_{\text {eff }}$, and a more efficient $3 \mathrm{du}$, such that the blue line is catching up in the upper panel of Fig. 12. Although the final $\log T_{\text {eff }}$ is about 0.1 dex lower than in the other case, $\mathrm{C} / \mathrm{O}$ at the surface is still slightly lower. As a consequence of the lower $T_{\text {eff }}$, mass loss is higher and the evolution stops earlier in the "variable opacities" case.

This effect of an initially shallower $T_{\text {eff-decrease as a con- }}$ sequence of carbon-enrichment of the envelope is more pronounced for lower overall metallicity, as can be inferred already from Figs. 11 and 12. We have verified this with a further test case at our lowest metallicity $\left(M=1.8 M_{\odot}, Z=0.0005\right)$. The reason is that with decreasing metallicity the opacity maximum for $\mathrm{C} / \mathrm{O}>1$ is shifting from $\log T \approx 3.4$ for $Z=0.02$ (Fig. 2) to $\log T \approx 3.35$ for $Z=0.0003$ (which is the table metallicity closest to that of the model, see Fig. 13), and that the C-rich opacities are no longer higher at $\log T \approx 3.5$. This effect is confirmed by Cristallo et al. (2007, their Fig. 4) for their $2 M_{\odot}$, $Z=0.0001$ model, calculated also with carbon-enhanced molecular opacities. As a consequence of the higher temperatures of Cenhanced models, mass loss is decreased and TP-AGB lifetimes prolonged. This figure also shows that when $\mathrm{C}$-enhancement is not taken into account at all, i.e. if $Z$ is kept constant, $T_{\text {eff }}$ is hardly decreasing at all. The same influence of an increasing C/O-ratio on the Rosseland mean opacity can be found in the data by Lederer \& Aringer (2009, their Fig. 2).

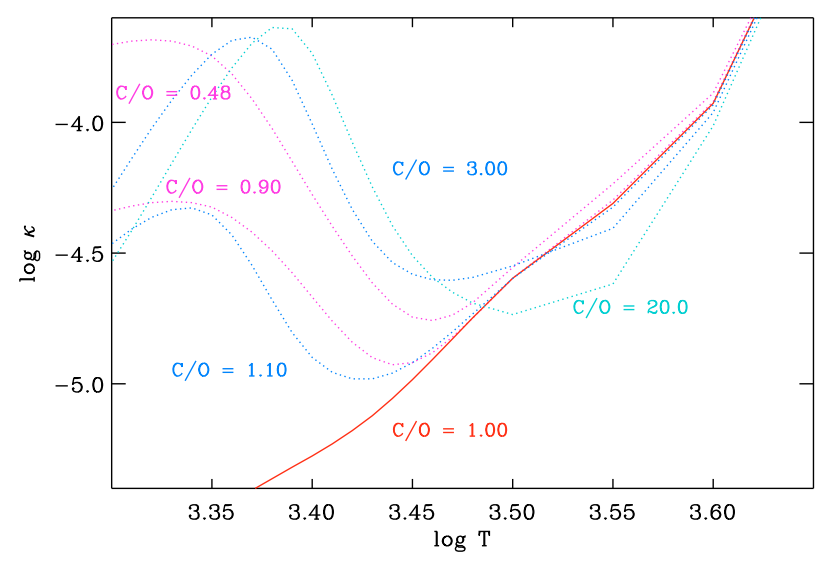

Fig. 13. As Fig. 2, but for $Z=0.0003$. The increase in $\kappa$ due to strong carbon enhancement has shifted to lower temperature.

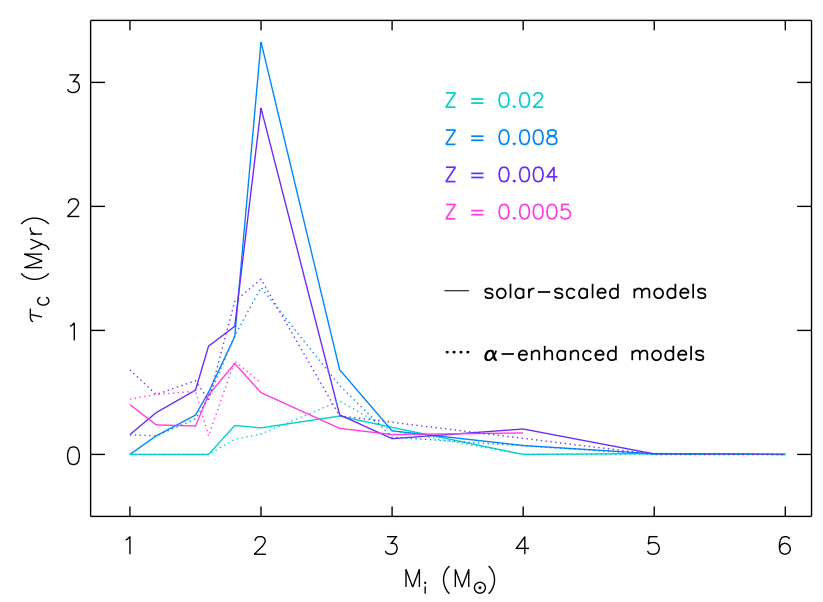

Fig. 14. Lifetimes of all our models as carbon stars. solar-scaled metal mixtures refer to the solid lines, $\alpha$-enhanced ones to to the dotted lines.

\subsection{Carbon-stars lifetimes}

The interaction between dredge-up, low-temperature opacities, and mass loss determines for what duration a stellar model is a C-star. Since our calculations differ from previous full AGB models in those aspects, we expect that carbon-star lifetimes will be modified considerably. We show in Fig. 14 the C-star lifetimes of our models for all 10 compositions. The efficiency of the $3 \mathrm{du}$, which rises with lower metallicity, leads to the pronounced peaks for $Z=0.004$ and $Z=0.008$, but the lowest metallicity models have a much smaller peak. The location of the lifetime peak shifts to lower initial mass with decreasing metallicity, although for $Z=0.02$ it is not very pronounced. Note that the $Z=0.04$ case is missing as for this metallicity our models do not turn into carbon stars (Table B.1).

Girardi \& Marigo (2007) have derived the C-star lifetime from star counts in clusters of both Magellanic clouds. Their Fig. 3 shows a comparison of these with synthetic AGB population predictions. They note that the carbon-star luminosity function could be reproduced successfully only since the work of Groenewegen \& de Jong (1993), but that even this model, as well as all previous ones underestimated the C-star lifetime peak for initial masses below $3 M_{\odot}$, and overestimated it for higher masses. Marigo (2002), introducing the C-variable molecular opacities managed to obtain an acceptable fit to the observed data. In Fig. 15 we compare the data by Girardi \& Marigo (2007) for the LMC with the predicted lifetimes of our own models 


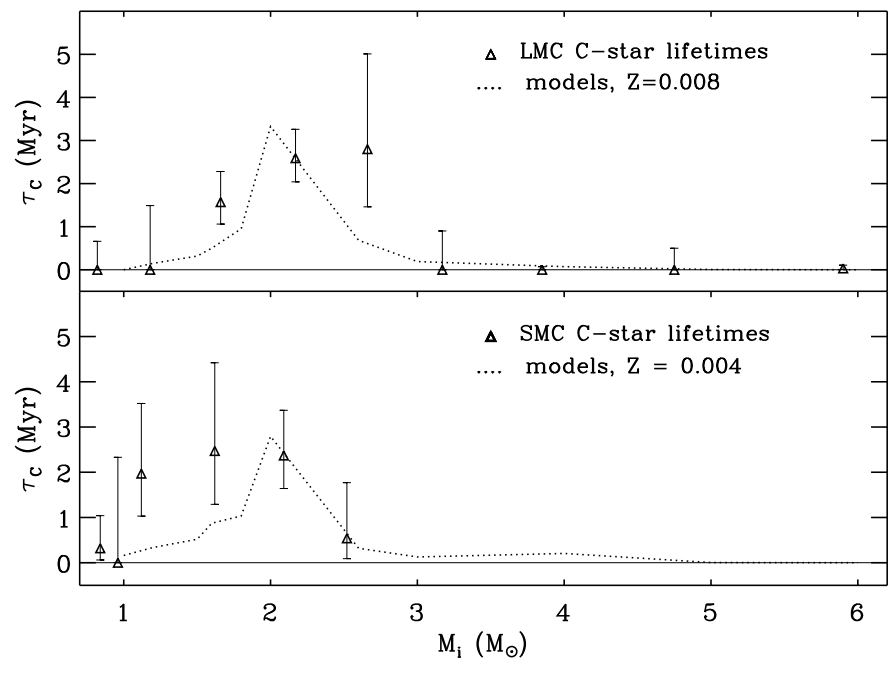

Fig. 15. Lifetimes of carbon stars, $\tau_{\mathrm{C}}$ in the LMC (upper panel) and SMC (lower panel). The observational data and error-bars are from the compilation by Girardi \& Marigo (2007), the dotted lines correspond to our model predictions for the two appropriate compositions with solarscaled metal ratios, as given in the figure.

with $Z=0.008$ (upper panel) and for the SMC with models with $Z=0.004$ (lower panel; both for solar-scaled metallicities). In both cases, but in particular for the LMC, the agreement is quite satisfying, although the peak width is too low for the SMC. Note also that the C-star lifetimes are much lower for initially $\alpha$ enhanced composition (Fig. 14, dotted lines). As we mentioned before this is simply a consequence of the initially lower $\mathrm{C} / \mathrm{O}$ ratio that requires more $3 \mathrm{du}$ episodes.

\subsection{Post-AGB evolution}

At the end of the AGB-phase, the models evolve to higher $T_{\text {eff }}$ with decreasing pulsation period. To date the largest set of postAGB tracks are still the 27 tracks by Vassiliadis \& Wood (1994) for the same metallicities as in Vassiliadis \& Wood (1993), and covering the post-AGB mass range from $0.558 M_{\odot}$ to $0.943 M_{\odot}$. These post-AGB models are in fact the continuation of the AGB-models of Vassiliadis \& Wood (1993), and constitute the very rare case of continuous evolutionary models that evolve from the main-sequence to the white dwarf cooling stage. In our case, as mentioned before, we encountered severe convergence problems for many models, which are similar to those reported, e.g., by Wood \& Faulkner (1986), Herwig (2005), Miller Bertolami \& Althaus (2006b), and Karakas \& Lattanzio (2007). From the 100 tracks evolved to the end of the AGB, or sufficiently close to it, only 60 could be followed through the postAGB phase. They are all from initial masses in the range of 1 to $2 M_{\odot}$. Half of them could be computed continuously, for the other half we had to "freeze" the models at the end of the AGB, remove the remaining envelope, and resume the full evolution at sufficiently higher temperature. In many cases this was successful only when $T_{\text {eff }} \approx 10^{4} \mathrm{~K}$, which is a standard definition for the start of the post-AGB evolution (Vassiliadis \& Wood 1994; Marigo et al. 2004). The time between the beginning departure from the AGB and this point is taken as the transition time $t_{\mathrm{tr}}$. We derive $t_{\mathrm{tr}}$ in those cases where we have continuous models as the time between the model for which the pulsation period has dropped to 100 days and the $T_{\text {eff }}=10^{4} \mathrm{~K}$ point. As a consequence of the restricted initial mass range for which we were able to follow the post-AGB evolution, post-AGB stellar masses ranged only between 0.5 and $0.6 M_{\odot}$. About one third of our models experience a late or very late TP (LTP or VLTP) during their horizontal crossing of the HRD respectively on the WD cooling track. The calculations were stopped when they had returned to the AGB for the second time. Such models had to be followed in each case separately to overcome the numerical difficulties.

Since our post-AGB grid is restricted in mass, we refer to Kitsikis (2008) for the detailed results. Here we summarize only a few key points. Of the 30 cases for which we could follow the complete evolution without intervention, 4 left the AGB as Heburners (defined as leaving the AGB through a pulse cycle phase below 0.15). Although this number is as low as that in Vassiliadis \& Wood (1994) and Bloecker (1995b), it cannot be compared directly, since in both these cases most He-burners originate from born-again stars, i.e. stars that experienced a (V)LTP. Including this fact, our fraction of He-burners would be higher. On the other hand, we can expect that models with higher initial mass, if we could follow them to the post-AGB, would reduce the fraction of He-burners. This is because our mass-loss description favors envelope ejection during the late TP-cycle only for lowmass stars, while for higher masses the mass loss rate remains high through a large fraction of the interpulse phase, too.

The transition times greatly depend upon the definition of the post-AGB phase and the termination of the final AGBsuperwind. Vassiliadis \& Wood (1994) begin the post-AGB evolution between temperatures of $3500 \mathrm{~K}$ and $5000 \mathrm{~K}$, while those by Bloecker (1995b) start between $6000 \mathrm{~K}$ and $7900 \mathrm{~K}$. Our definition of the $100 \mathrm{~d}$ pulsation period leads to an earlier post-AGB start, corresponding to $T_{\text {eff }} \approx 3800, \ldots 5400 \mathrm{~K}$. Similarly, the post-AGB mass loss description influences the transition speed. Vassiliadis \& Wood (1994) rather abruptly switch to radiationdriven winds, which for $T_{\mathrm{eff}}<10000$, the end of the transition phase, is lower than our adopted Reimers-type wind (Eq. (3)), which is similar to the approach by Bloecker (1995b). As a result, $t_{\text {tr }}$ ranges from a few hundred to about 2000 yrs for most of our models, with a slight tendency to increase for lower masses and higher metallicities (up to $\approx 10000$ yrs for $Z=0.04$ ). Two He-burner post-AGB models, both with $Z=0.04$ and of initial mass $1 M_{\odot}$ (solar and $\alpha$-enhanced cases) are outstanding and reach 50000 years. The overlap in post-AGB mass with Vassiliadis \& Wood (1994) is very restricted; their $t_{\mathrm{tr}}$ in the mass range in common with us is up to ten times longer. According to Schönberner \& Steffen (2007) post-AGB stars have $T_{\text {eff }} \gtrsim$ $5000 \mathrm{~K}$ and transition times around $1000 \mathrm{yrs}$, as inferred from hydrodynamical studies. Our models thus show a reasonable agreement with these results.

Two further timescales of the post-AGB evolution are of interest. The first one is the time until the radiation-driven hot wind of Eq. (6) sets in (technically the point, when it is larger than the Reimers wind of Eq. (3)). Since our post-AGB mass loss description is very similar to that by Bloecker (1995a), we can compare this timescale $t_{1}$, but only for the mass in common, $M=0.6 M_{\odot}$, where his $t_{1}$ is $1700 \mathrm{yrs}$ while ours is $t_{1} \approx 500 \mathrm{yrs}$. This is close to that by Bloecker (1995a) for $M=0.625 M_{\odot}$, who finds an increase in $t_{1}$ with decreasing mass, strongest for the lowest masses, which we confirm.

The second timescale is the total crossing time, $t_{\mathrm{cr}}$, taken from $T_{\text {eff }}=10^{4} \mathrm{~K}$ to the turn-around point at the "knee" of the post-AGB evolution, typically around $T_{\text {eff }} \approx 10^{5} \mathrm{~K}$. In Fig. 16 we compare our results with those by Vassiliadis \& Wood (1994). Our values for $t_{\text {tr }}$ are lower because of the faster early evolution on the post-AGB. However, some basic features are similar: A strong increase in $t_{\mathrm{cr}}$ with decreasing mass, higher 


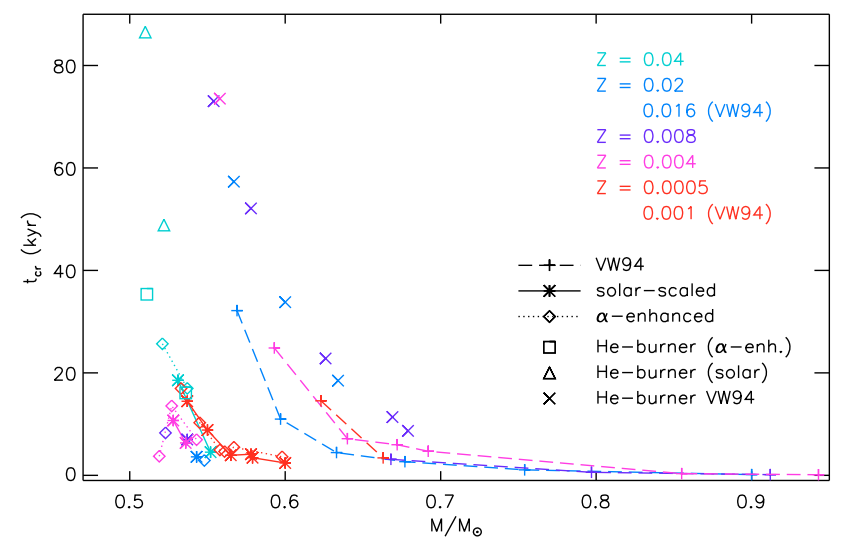

Fig. 16. Post-AGB crossing times (defined in the text) for our models, both for solar-scaled (stars) and $\alpha$-enhanced (diamonds) mixtures, indicated by colours. For comparison, the results by Vassiliadis \& Wood (1994) are also plotted (crosses). He-burner post-AGB stars are given by the larger open symbols, not connected by lines.

Table 5. Born-again times $t_{\mathrm{ba}}$ for models experiencing a (V)LTP.

\begin{tabular}{cccccc}
\hline \hline$M_{\text {ZAMS }}\left(M_{\odot}\right)$ & Comp. & $M_{\text {T4 }}\left(M_{\odot}\right)^{a}$ & $t_{\text {ba }}(\mathrm{yr})$ & $\log L_{\max }^{b}$ & $\Delta t(\mathrm{yr})^{c}$ \\
\hline 1.6 & III & 0.541 & 189 & 9.386 & $10^{-7 /-2}$ \\
1.0 & V & 0.531 & 240 & 10.05 & $10^{-7 /-2}$ \\
1.6 & V & 0.537 & 56 & 9.959 & $10^{-7 /-2}$ \\
1.2 & VI & 0.531 & 292 & 6.079 & $10^{-5 /-2}$ \\
1.5 & VI & 0.537 & 302 & 6.674 & $10^{-5 /-2}$ \\
1.6 & VI & 0.536 & 307 & 7.006 & $10^{-5 /-2}$ \\
1.5 & VII & 0.536 & 200 & 10.34 & $10^{-4}$ \\
1.2 & VIII & 0.538 & 254 & 6.559 & $10^{-4 /-1}$ \\
1.6 & VIII & 0.519 & 305 & 6.961 & $10^{-6 /-2}$ \\
\hline
\end{tabular}

${ }^{a}$ Initial post-AGB mass; ${ }^{b}$ maximum $\mathrm{H}$-luminosity during the late TP; ${ }^{c}$ minimum time-step(s).

values for lower metallicities at the same mass, longer crossing times for He-burner, and a majority of values below or around $10^{4}$ yrs.

A summary of the various timescales is given in Tables C.1 and C.2. The meaning of the various columns in these tables is as follows: (1) initial stellar mass on ZAMS; (2) $T_{\text {eff }}$ at which post-AGB evolution starts; a star indicates that this is not the point with a pulsation period of $100 \mathrm{~d}$, but rather the first model after "frozen-in" envelope stripping; (3) stellar mass at $T_{\text {eff }}=10^{4} \mathrm{~K}$; (4) transition time between the post-AGB start and $T_{\text {eff }}=10^{4} \mathrm{~d}$ (this is a lower limit for the cases marked by a star); (5) time $t_{1}$ between beginning of post-AGB evolution and onset of radiation-driven wind; (6) time $t_{\mathrm{cr}}$ for crossing of HRD; (7) H- or He-burner, or both, if a LTP or VLTP (indicated by Column 8) happens; (9) end of the calculations: "WD" indicates that the WD cooling track was reached; otherwise the computations were ended due to numerical problems in the phase indicated.

Since some of our models experience a LTP or VLTP (see Tables C.1 and C.2, from which also the initial post-AGB mass $M_{\mathrm{T} 4}$ is taken), we provide in Table 5 the "born-again times" $t_{\mathrm{ba}}$, taken as the time between the maximum hydrogen-luminosity after a (very) late TP has occurred and the moment the star arrives again at $\log T_{\text {eff }}=3.8$ (Miller Bertolami \& Althaus 2007). Observationally, (e.g. Asplund 1999, for Sakurai's object), $t_{\text {ba }}$ is as short as a few years, while theoretical models (e.g. Herwig et al. 1999; Herwig 2001) predict several hundred years. However, Miller Bertolami et al. (2006) obtained bornagain times of 5-10 years by using an extremely fine time resolution. As was pointed out by Herwig (2001), care has to be taken with regard to the shortest time steps. The reason is that the mixing length theory is applicable only to stationary convective situations. The adjustment of convection to changing conditions is not considered. Therefore, time-steps shorter than typical convective turn-over times are inconsistent with the assumptions of mixing length theory, if convective layers are changing quickly. This may be the case during the fast rise of temperature due to violent nuclear burning, as is the case in the hot hydrogen burning regions of a born-again star. The typical turn-over times in this phase is of order $5 \mathrm{~min}$, or $10^{-5}$ years. Consequently, timesteps should be longer than this, and therefore Miller Bertolami et al. (2006) set the minimum time-step allowed in their calculations to this value. For some of our models in Table 5, however, we had to switch to even smaller time-steps to achieve numerical convergence. Although this happened typically only for the very short period around the H-luminosity maximum, and for a duration of order 1 hour (e.g. for the model with initially $1.6 M_{\odot}$ and $M_{\mathrm{T} 4}=0.558$ the time-step was $10^{-7}$ years for a total duration of $2.3 \mathrm{~h}$ ), these models have to be taken with care. The total born-again time for all of the cases shown is of the order of several hundred years, in agreement with Herwig (2001). In this paper it was also demonstrated that reducing the velocity of convective elements, shorter born-again times can be achieved, while Miller Bertolami et al. (2006) found born-again times of 5-10 years when going to the lower limit of acceptably small time-steps. While we in principle confirm this model behaviour, we had to go to even lower time-steps for the whole evolution past the onset of the VLTP and therefore, as the referee correctly pointed out, such models should be considered as being unphysical.

\section{Discussion}

Our grid of stellar models evolving into, through, and past the Asymptotic Giant Branch phase covers a wide range of metallicities, from $Z=0.0005$ to $Z=0.04$ and the two standard metal distributions, solar-scaled and $\alpha$-enhanced. The physical input - equation of state, opacities, nuclear reaction rates - are completely up-to-date. Both features are new for grids of AGB models. The masses followed the range from 1.0 to $6.0 M_{\odot}$. Our grid should provide valuable input for population studies and for synthetic AGB models, although we have not developed new analytical functions to represent key quantities, such as the core-mass - luminosity relation, or the interpulse times. Previous relations (Wagenhuber \& Groenewegen 1998; Izzard et al. 2004) appear to be good first-order approximations, which could be tuned to our models without large changes.

We specifically concentrated on a consistent treatment of carbon enrichment of the envelope due to the third dredge-up. Carbon enrichment is taken into account in the opacities, which have been available not only for various $(X, Y, Z)$-mixtures, but also for changes in the C/O-ratio. To this end new lowtemperature molecular opacity tables were calculated and included in the stellar evolution program. The increase in carbon leads to lower effective temperature, mainly by the increase in total metallicity. We found that at same metallicity, an increase in $\mathrm{C} / \mathrm{O}$ can initially lead to lower opacities and thus higher effective temperatures as compared to a solar-scaled mixture. At $\mathrm{C} / \mathrm{O} \gtrsim 2$ finally higher opacities are reached.

For the crucial mass loss of highly evolved AGB stars we used an empirical mass loss formula for oxygen stars (van Loon et al. 2005) and a theoretical one for carbon stars 
(Wachter et al. 2002). They are both similar in the order of magnitude of the mass loss and used only if the pulsation period is above $400 \mathrm{~d}$. The switching-on of a strong mass loss from an (enhanced) Reimers wind happens quite suddenly at a pulsation period of $400 \mathrm{~d}$, with a definite decrease in effective temperature. This is to be considered as a consequence of our specific mass loss description. Carbon-enhancement together with increasing opacities and decreasing effective temperature lead to a strong mass loss, which eventually terminates in a strong "superwind".

Third dredge-up is obtained by assuming overshooting (implemented by a diffusion approach), the extent of which is set by the free parameter $f$ of this prescription (Eq. (2)). We used a numerical value of 0.016 obtained from other, independent calibrations (open clusters, upper main-sequence). Although a very similar value has also been used in earlier AGB models (Herwig et al. 1999; Herwig 2000), specific comparison with the results of nuclear processing indicate that it may vary within AGB stars: being possibly smaller in the pulse-driven convection zone (Lugaro et al. 2003), and larger at the bottom of the convective envelope (Herwig et al. 2003). In spite of these hints we decided to refrain from varying the overshooting parameter. Nevertheless, the result of the third dredge-up, obtained with this prescription and lifetimes for carbon-stars are in good agreement with observations. Such agreement has been reached before only with tunable synthetic models.

On the other hand, our predicted initial-final mass relation, though overall in very good agreement with a recent empirical determination, shows that the hydrogen-free core grows less than observed for the lowest initial stellar masses. This is due to our application of overshooting to all convective boundaries. A reduction of overshooting at the base of the pulse-driven convection zone seems to be supported.

A severe problem - not specific to our calculations - are the convergence failures for highly evolved AGB stars. In particular for the higher masses and lower metallicities they partially prevent useful calculations. They are physically connected to a dominance of radiation pressure in the lower convective envelope (Wood \& Faulkner 1986), which also leads to non-physical, super-sonic convective velocities (Wagenhuber 1996) within the mixing-length theory. These convergence problems appear in many modern AGB calculations, such as Karakas (2003), which has been the most extensive and modern grid so far. A proper treatment, guided by hydrodynamical calculations, is urgently asked for. Since we did not use any of the "recipes" to somehow bypass this critical phase, the number of reasonably complete AGB models is $\approx 100$, out of the 110 models our grid in total comprises. For the post-AGB phase, the number even reduces to 60 , out of which only 30 could be followed from the ZAMS to the WD stage without interruption. The remaining 30 cases were obtained by artificially stripping off the residual envelope in the early AGB-transition phase. All these cases correspond to an initial mass of up to $2 M_{\odot}$ and a post-AGB mass below $0.60 M_{\odot}$. These models constitute a useful extension of previous post-AGB models by Vassiliadis \& Wood (1994) and Bloecker (1995a) to lower post-AGB masses.

Since we aimed at treating the effect of carbon-enrichment of the envelope as consistently as possible, it is interesting to compare the effective temperatures of our models with those obtained with the synthetic models by Marigo \& Girardi (2007), who had a similar fully consistent treatment of AGB evolution in mind, and calibrated their synthetic models to observed carbon-star luminosity functions. We specifically looked into their model of $1.8 M_{\odot}$ and $Z=0.008$ (shown in Fig. 5 of that paper). In fact, the $T_{\text {eff }}$ evolution during the AGB-phase is very similar: Both models start at $\log T_{\text {eff }} \approx 3.6$ in the early interpulse phases and then become cooler down to $\log T_{\text {eff }} \approx 3.4$ and below during the last TP. However, our model shows a more gradual decrease because of the better resolution of $\mathrm{C} / \mathrm{O}$-variations in the WSU molecular opacities. There are, of course, differences, as well. Our model experiences only 9 TPs (plus 1 estimated final $\mathrm{TP}$ ), while theirs has 32 , with $\mathrm{C} / \mathrm{O}>1$ reached at TP 19 . The final C/O-ratio is close to 3. Our model ends at $\mathrm{C} / \mathrm{O}=2.004$, and turns into a C-star after TP 4. This is due to the different dredgeup efficiencies and mass loss descriptions. A similar agreement is found for the $M=4 M_{\odot}$ model, except that our model does not experience $\mathrm{HBB}$ and therefore the increase in $T_{\text {eff }}$ due to a decrease in $\mathrm{C} / \mathrm{O}$ is not taking place. Before that event, during the initial $3 \mathrm{du}$, both models also show a similar $T_{\text {eff-development. }}$ Marigo \& Girardi (2007) emphasize that their improved treatment of molecular opacities leads to much better agreement with observed colors of AGB stars (Marigo et al. 2008). Together with the fact that our full AGB models are the first to reproduce the carbon-star lifetimes as derived by Girardi \& Marigo (2007), we conclude that overall our full models agree well with these calibrated and successful synthetic models, although they were computed with pre-defined physical input.

In the present work, a detailed investigation of nucleosynthesis and chemical yields was ignored (see Karakas 2003, for such details). However, we can confirm that our models display the occurrence of ${ }^{13} \mathrm{C}$-pockets as a consequence of $3 \mathrm{du}$ and convective mixing. Therefore, the necessary precondition for $s$ process nucleosynthesis is given, but a detailed analysis with a post-processing network is still needed.

We have used the most up-to-date and self-consistent physical approach to full AGB models. To our surprise, our models often agree better with the older Vassiliadis \& Wood (1993) models than with the newer ones by Karakas. These two sets were actually calculated with the same code, but in different versions, separated by a decade of development. We think that this indicates that the numerical and technical aspects of implementing the relevant physics for AGB-stars is still an important factor, such that future work in this direction is as important as further improvements in the physics itself.

Acknowledgements. We have presented in this paper mainly the results of the Ph.D. Thesis by Agis Kitsikis, to whom we are obliged for allowing us to use his calculations. We thank P. Marigo for making available her molecular opacities to us, and M. Miller Bertolami for model comparisons, additional calculations, and helpful discussions. The (anonymous) referee provided an exceptionally expert and helpful review which corrected several misunderstandings on our side, and for which we are very grateful.

\section{References}

Adelberger, E. G., Austin, S. M., Bahcall, J. N., et al. 1998, Rev. Mod. Phys., 70, 1265

Arndt, T. U., Fleischer, A. J., \& Sedlmayr, E. 1997, A\&A, 327, 614

Asplund, M. 1999, in Asymptotic Giant Branch Stars, ed. T. Le Bertre, A. Lebre, \& C. Waelkens, IAU Symp., 191, 481

Bloecker, T. 1995a, A\&A, 299, 755

Bloecker, T. 1995b, A\&A, 297, 727

Cassisi, S., Salaris, M., \& Irwin, A. W. 2003, ApJ, 588, 862

Caughlan, G. R., Fowler, W. A., Harris, M. J., et al. 1985, Atomic Data and

Nuclear Data Tables, 32, 197

Coelho, P., Bruzual, G., Charlot, S., et al. 2007, MNRAS, 382, 498

Cordier, D., Pietrinferni, A., Cassisi, S., et al. 2007, AJ, 133, 468

Cristallo, S., Straniero, O., Lederer, M. T., et al. 2007, ApJ, 667, 489

Cristallo, S., Straniero, O., Gallino, R., et al. 2009, ApJ, 696, 797

Denissenkov, P. A., \& Tout, C. A. 2003, MNRAS, 340, 722

Dotter, A., Chaboyer, B., Jevremović, D., et al. 2008, ApJS, 178, 89

Ferguson, J. W., \& Dotter, A. 2008, in IAU Symp., 1

Ferguson, J. W., Alexander, D. R., Allard, F., et al. 2005, ApJ, 623, 585

Fleischer, A. 1994, Ph.D. Thesis, Technischen Universität Berlin 
Fleischer, A. J., Gauger, A., \& Sedlmayr, E. 1992, A\&A, 266, 321

Formicola, A., Imbriani, G., Costantini, H., et al. 2004, Phys. Lett. B, 591, 61 Freytag, B., Ludwig, H.-G., \& Steffen, M. 1996, A\&A, 313, 497

Fynbo, H. O. U., Diget, C. A., Bergmann, U. C., et al. 2005, Nature, 433, 136 Girardi, L., \& Marigo, P. 2007, A\&A, 462

Girardi, L., Bressan, A., Bertelli, G., et al. 2000, A\&A, 141, 371

Grevesse, N., \& Noels, A. 1993, Phys. Scr. T, 47, 133

Grevesse, N., \& Sauval, A. 1998, Space Sci. Rev., 85, 161

Groenewegen, M. A. T., \& de Jong, T. 1993, A\&A, 267, 410

Groenewegen, M., \& Whitelock, P. 1996, MNRAS, 281, 1347

Herwig, F. 2000, A\&A, 360, 952

Herwig, F. 2001, ApJ, 554, L71

Herwig, F. 2004a, ApJ, 605, 425

Herwig, F. 2004b, ApJS, 155, 651

Herwig, F. 2005, ARA\&A, 43, 435

Herwig, F., Bloecker, T., Schönberner, D., et al. 1997, A\&A, 324, L81

Herwig, F., Blöcker, T., Langer, N., et al. 1999, A\&A, 349, L5

Herwig, F., Langer, N., \& Lugaro, M. 2003, ApJ, 593, 1056

Herwig, F., Freytag, B., Fuchs, T., et al. 2007, in Why Galaxies Care About AGB Stars: Their Importance as Actors and Probes, ed. F. Kerschbaum, C. Charbonnel, \& R. F. Wing, ASP Conf. Ser., 378, 43

Iben, Jr., I., \& Truran, J. W. 1978, ApJ, 220, 980

Iglesias, C., \& Rogers, F. 1996, ApJ, 464, 943

Izzard, R. G., Tout, C. A., Karakas, A. I., et al. 2004, MNRAS, 350, 407

Karakas, A. I. 2003, Ph.D. Thesis, Monash University, Australia

Karakas, A. I., \& Lattanzio, J. C. 2007, ArXiv e-prints, 708

Kitsikis, A. 2008, Ph.D. Thesis, Ludwig-Maximilians-Universität München

Kunz, R., Fey, M., Jaeger, M., et al. 2002, ApJ, 567, 643

Langer, N., Heger, A., Wellstein, S., et al. 1999, A\&A, 346, L37

Lederer, M. T., \& Aringer, B. 2008, in Evolution and Nucleosynthesis in AGB

Stars, ed. R. Guandalini, S. Palmerini, \& M. Busso, AIP Conf. Ser., 1001, 11

Lederer, M. T., \& Aringer, B. 2009, A\&A, 494, 403

Liu, M. C., Charlot, S., \& Graham, J. R. 2000, ApJ, 543, 644

Liu, Y., Liu, X.-W., Barlow, M. J., et al. 2004, MNRAS, 353, 1251

Lugaro, M., Herwig, F., Lattanzio, J. C., Gallino, R., \& Straniero, O. 2003, ApJ, 586, 1305

Marigo, P. 2002, A\&A, 387, 507

Marigo, P., \& Girardi, L. 2007, A\&A, 469, 239

Marigo, P., Bressan, A., \& Chiosi, C. 1996, A\&A, 313, 545

Marigo, P., Girardi, L., Weiss, A., Groenewegen, M. A. T., \& Chiosi, C. 2004, A\&A, 423, 995

Marigo, P., Girardi, L., Bressan, A., et al. 2008, A\&A, 482, 883

Mattsson, L., Wahlin, R., Höfner, S., et al. 2008, A\&A, 484, L5

Meissner, F., \& Weiss, A. 2006, A\&A, 456, 1085

Mihalas, D., Däppen, W., \& Hummer, D. G. 1988, ApJ, 331, 815

Miller Bertolami, M. M., \& Althaus, L. G. 2006a, A\&A, 454, 845
Miller Bertolami, M. M., \& Althaus, L. G. 2006b, A\&A, 454, 845

Miller Bertolami, M. M., \& Althaus, L. G. 2007, MNRAS, 380, 763

Miller Bertolami, M. M., Althaus, L. G., Serenelli, A. M., et al. 2006, A\&A, 449, 313

Ostlie, D. A., \& Cox, A. N. 1986, ApJ, 311, 864

Paczyński, B. 1970, Acta Astron., 20, 47

Pauldrach, A., Puls, J., Kudritzki, R.-P., Mendez, R., \& Heap, S. 1988, A\&A, 207, 123

Péquignot, D., Zijlstra, A. A., Walsh, J., et al. 2000, in ed. S. J. Arthur, N. S. Brickhouse, \& J. Franco, 9, Rev. Mex. Astron. Astrofis Conf. Ser., 220

Pietrinferni, A., Cassisi, S., Salaris, M., et al. 2004, ApJ, 612, 168

Raimondo, G., Brocato, E., Cantiello, M., et al. 2005, AJ, 130, 2625

Reimers, D. 1975, Mem. Soc. Roy. Sci. Liège, 8, 369

Renzini, A., \& Voli, M. 1981, A\&A, 94, 175

Rogers, F., Swenson, F., \& Iglesias, C. 1996, ApJ., 456, 902

Salaris, M., Serenelli, A., Weiss, A., et al. 2009, ApJ, 692, 1013

Scalo, J. M., \& Ulrich, R. K. 1975, ApJ, 200, 682

Schlattl, H., Cassisi, S., Salaris, M., et al. 2001, ApJ, 559, 1082

Schönberner, D., \& Steffen, M. 2007, in Why Galaxies care about AGB Stars: their importance as actors and probes, ed. F. Kerschbaum, C. Charbonnel, \& R. F. Wing, ASP Conf. Ser., 378, 343

Seaton, M. J. 2007, MNRAS, 382, 245

Seaton, M. J., Zeippen, C. J., Tully, J. A., et al. 1992, Rev. Mex. Astron. Astrofis, 23, 19

Sedlmayr, E., \& Winters, J. 1997, in Stellar Atmospheres: Theory and Observations, ed. J. P. de Greve, R. Blomme, \& H. Hensberge, Lect. Notes Phys. (Berlin: Springer Verlag), 497, 89

Serenelli, A., \& Weiss, A. 2005, A\&A, 442, 1041

van Loon, J., Cioni, M.-R., Zijlstra, A., et al. 2005, A\&A, 438, 273

Vassiliadis, E., \& Wood, P. R. 1993, ApJ, 413, 641

Vassiliadis, E., \& Wood, P. R. 1994, ApJS, 92, 125

Ventura, P., Zeppieri, A., Mazzitelli, I., et al. 1998, A\&A, 334, 953

Wachter, A., Schröder, K.-P., Winters, J., Arndt, T., \& Sedlmayr, E. 2002, A\&A, 384,452

Wachter, A., Winters, J. M., Schröder, K.-P., et al. 2008, A\&A, 486, 497

Wagenhuber, J. 1996, Ph.D. Thesis, Techn. Univ. München

Wagenhuber, J., \& Groenewegen, M. A. T. 1998, A\&A, 340, 183

Wagenhuber, J., \& Weiss, A. 1994, A\&A, 286, 121

Wallerstein, G., \& Knapp, G. 1998, ARA\&A, 36, 369

Weidemann, V. 2000, A\&A, 363, 647

Weiss, A., \& Schlattl, H. 2008, Ap\&SS, 316, 99

Weiss, A., Serenelli, A., Kitsikis, A., Schlattl, H., \& Christensen-Dalsgaard, J. 2005, A\&A, 441, 1129

Werner, K., \& Herwig, F. 2006, PASP, 118, 183

Winters, J., Fleischer, A., Le Bertre, T., et al. 1997, A\&A, 326, 305

Wood, P. R., \& Faulkner, D. J. 1986, ApJ, 307, 659 
A. Weiss and J. W. Ferguson: AGB model grid, Online Material p 1

\section{Appendix A: Evolution up to the first TP}

Table A.1. Solar-scaled metallicity models from the ZAMS until the $1^{\text {st }} \mathrm{TP}$.

\begin{tabular}{|c|c|c|c|c|c|c|c|c|c|c|c|c|c|}
\hline \multicolumn{7}{|c|}{$\overline{Z Z=0.02 / \text { solar }}$} & \multicolumn{7}{|c|}{$\overline{Z Z=0.008 / \text { solar }}$} \\
\hline$M_{\text {ZAMS }}$ & $t_{\mathrm{MS}}{ }^{a}$ & $t_{\mathrm{RGB}}$ & $t_{\mathrm{He}-\mathrm{b}}$ & $t_{\mathrm{EAGB}}$ & $M_{\mathrm{tot}}(1)^{b}$ & $M_{\mathrm{c}}(1)^{c}$ & $M_{\mathrm{ZAMS}}$ & $t_{\mathrm{MS}}$ & $t_{\mathrm{RGB}}$ & $t_{\mathrm{He}-\mathrm{b}}$ & $t_{\mathrm{EAGB}}$ & $M_{\mathrm{tot}}(1)$ & $M_{\mathrm{c}}(1)$ \\
\hline 1.0 & 8616.2 & 3350.1 & 110.17 & 12.971 & 0.709 & 0.501 & 1.0 & 6751.9 & 2521.0 & 104.48 & 10.369 & 0.742 & 0.513 \\
\hline 1.2 & 4473.9 & 1555.6 & 106.47 & 12.368 & 0.981 & 0.509 & 1.2 & 3600.5 & 1182.0 & 97.963 & 10.474 & 0.999 & 0.521 \\
\hline 1.5 & 2560.8 & 242.28 & 108.73 & 11.645 & 1.353 & 0.511 & 1.5 & 2097.9 & 193.39 & 99.367 & 9.8133 & 1.366 & 0.521 \\
\hline 1.6 & 2091.5 & 172.58 & 110.80 & 12.243 & 1.476 & 0.508 & 1.6 & 1725.1 & 137.98 & 100.87 & 11.363 & 1.490 & 0.516 \\
\hline 1.8 & 1465.8 & 99.847 & 130.85 & 13.305 & & 0.496 & 1.8 & 1223.8 & 78.851 & 128.10 & 11.802 & 1.637 & 0.497 \\
\hline 2.0 & 1075.0 & 58.364 & 177.34 & 15.417 & & 0.4 & 2.0 & 900.54 & 32.110 & 236.41 & 14.571 & 1.941 & 0.464 \\
\hline 2.6 & 510.25 & 12.302 & 140.08 & 10.175 & & & 2.6 & 448.03 & 9.9558 & 106.62 & 6.7430 & 06 & 0.569 \\
\hline 3.0 & 34 & 7.1561 & 83.500 & 5.7 & & & 3.0 & 24 & 5.9283 & 60.199 & 3.7127 & 63 & 0.672 \\
\hline 4.0 & & 2.7018 & 28.711 & & & & 4.0 & & 2.3324 & 23.405 & 66 & 12 & 801 \\
\hline 5.0 & 92 & 1.3843 & 13.577 & 0.9 & & & 5.0 & & 1.2398 & 84 & 29 & 76 & 0.868 \\
\hline 6.0 & 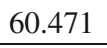 & 0.8147 & 8.1598 & & 7 & 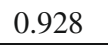 & 6.0 & 76 & 0.7491 & 7.7504 & 0.4100 & 5.711 & 0.991 \\
\hline \multicolumn{7}{|c|}{$\overline{Z Z=0.04 / \text { solar }}$} & \multicolumn{7}{|c|}{$\overline{Z Z=0.004 / \text { solar }}$} \\
\hline$M_{\text {ZAMS }}$ & $t_{\mathrm{MS}}$ & $t_{\mathrm{RGB}}$ & $t_{\mathrm{He}-\mathrm{b}}$ & & $M_{\mathrm{tot}}(1)$ & $M_{\mathrm{c}}(1)$ & $M_{\text {ZAMS }}$ & $t_{\mathrm{MIS}}$ & $t_{\mathrm{RGB}}$ & $t_{\mathrm{He}-\mathrm{b}}$ & $t_{\mathrm{EAGB}}$ & $M_{\mathrm{tot}}(1)$ & $M_{\mathrm{c}}(1)$ \\
\hline 1.0 & 8648.4 & 3974.3 & 119.31 & 14.213 & 0.682 & 0.504 & 1.0 & 5771.4 & 2050.5 & 92.852 & 10.965 & 0.776 & 0.517 \\
\hline 1.2 & 4788.6 & 1527.4 & 119.81 & 12.305 & 0.963 & 0.513 & 1.2 & 3141.2 & 966.74 & 94.451 & 9.3231 & 1.026 & 0.525 \\
\hline 1.5 & 2697.1 & 231.58 & 122.80 & 12.425 & 1.349 & 0.513 & 1.5 & 1847.2 & 164.02 & 94.336 & 9.2462 & 1.383 & 0.527 \\
\hline 1.6 & 2196.0 & 167.42 & 124.74 & 14.042 & 1.476 & 0.510 & 1.6 & 1525.1 & 117.01 & 103.22 & 9.0807 & 1.505 & 0.521 \\
\hline 1.8 & 1527.7 & 96.664 & 158.38 & 13.966 & 1.611 & 0.496 & 1.8 & 1088.4 & 65.247 & 131.73 & 11.283 & 1.672 & 0.499 \\
\hline 2.0 & 1108.6 & 54.286 & 226.81 & 17.353 & 1.903 & 0.481 & 2.0 & 812.67 & 27.464 & 199.22 & 11.361 & 1.935 & 0.488 \\
\hline 2.6 & 509.95 & 13.146 & 140.91 & 10.013 & 2.518 & 0.528 & 2.6 & 410.03 & 8.5853 & 82.059 & 4.0737 & 2.472 & 0.639 \\
\hline 3.0 & 337.90 & 7.6362 & 83.253 & 6.2347 & 2.893 & & 3.0 & 286.74 & 5.2255 & 50.584 & 2.3417 & 2.834 & 0.744 \\
\hline 4.0 & 151.95 & 2.8971 & 29.886 & 2.1740 & 3.826 & 0.7 & 4.0 & 145.00 & 2.1178 & 20.483 & 1.1477 & 3.801 & 0.809 \\
\hline 5.0 & 84.428 & 1.4122 & 13.929 & 1.0935 & 4.791 & & 5.0 & 88.228 & 1.1468 & 11.488 & 0.5852 & 4.775 & 0.894 \\
\hline 6.0 & 53.949 & 0.8091 & 7.9334 & 0.5929 & 5.651 & 0.893 & 6.0 & 60.070 & 0.7068 & 7.5367 & 0.3372 & 5.700 & 1.060 \\
\hline
\end{tabular}

\begin{tabular}{ccccccc}
\hline \hline \multicolumn{7}{c}{$Z=0.0005 /$ solar } \\
\hline$M_{\text {ZAMS }}$ & $t_{\mathrm{MS}}$ & $t_{\text {RGB }}$ & $t_{\mathrm{He}-\mathrm{b}}$ & $t_{\mathrm{EAGB}}$ & $M_{\text {tot }}(1)$ & $M_{\mathrm{c}}(1)$ \\
\hline 1.0 & 5041.2 & 1262.2 & 83.219 & 9.4308 & 0.847 & 0.523 \\
1.2 & 2662.3 & 727.55 & 86.164 & 7.4351 & 1.078 & 0.537 \\
1.5 & 1560.7 & 158.70 & 73.039 & 6.7829 & 1.351 & 0.570 \\
1.6 & 1278.6 & 104.02 & 87.687 & 6.7574 & 1.521 & 0.561 \\
1.8 & 912.34 & 46.277 & 147.74 & 6.5325 & 1.701 & 0.551 \\
2.0 & 682.51 & 22.101 & 127.21 & 5.6236 & 1.906 & 0.584 \\
2.6 & 351.50 & 7.1310 & 59.160 & 2.4622 & 2.464 & 0.732 \\
3.0 & 250.82 & 4.4426 & 39.685 & 1.6650 & 2.857 & 0.780 \\
4.0 & 132.50 & 1.8581 & 17.728 & 0.8079 & 3.867 & 0.848 \\
5.0 & 83.085 & 1.0331 & 9.9502 & 0.4567 & 4.875 & 0.937 \\
6.0 & 57.746 & 0.6695 & 6.3498 & 0.4317 & 4.208 & 1.128 \\
\hline
\end{tabular}

${ }^{a}$ All times are given in $10^{6} \mathrm{yrs} ;{ }^{b}$ total mass at $1^{\text {st }} \mathrm{TP}$ in solar unit; ${ }^{c}$ core mass at $1^{\text {st }} \mathrm{TP}$. 
A. Weiss and J. W. Ferguson: AGB model grid, Online Material $p 2$
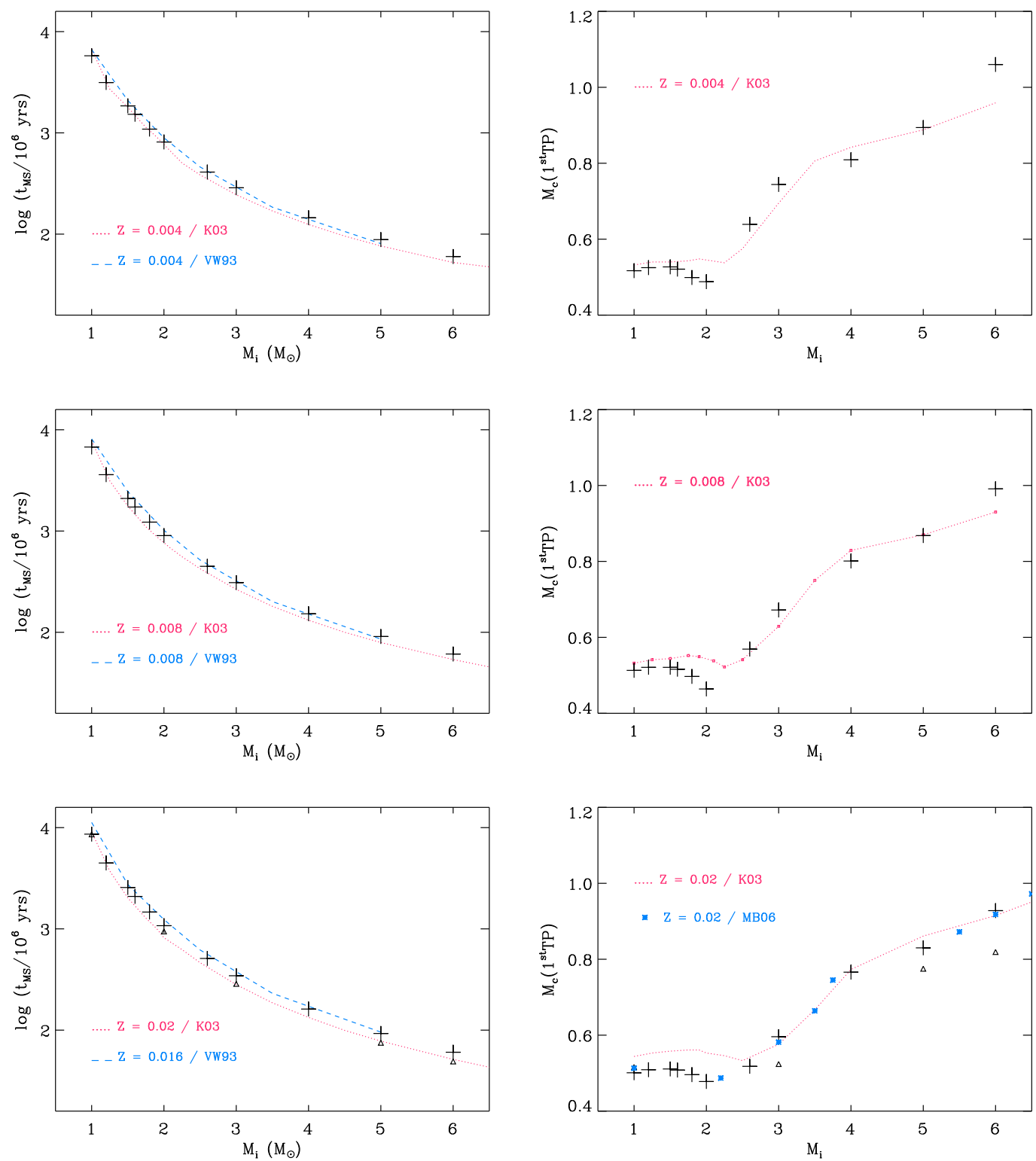

Fig. A.1. Main sequence lifetimes (left) and core mass at the first thermal pulse (right) for three chemical compositions with $Z=0.004,0.008,0.02$ (from top to bottom) of our models (crosses) in comparison with literature values from Karakas (2003, K03), Vassiliadis \& Wood (1993, VW93), and Miller-Bertolami (private communication, 2008, MB08). 
A. Weiss and J. W. Ferguson: AGB model grid, Online Material p 3

Table A.2. Same as for Table A.1 but for the $\alpha$-enhanced metallicity models.

\begin{tabular}{|c|c|c|c|c|c|c|c|c|c|c|c|c|c|}
\hline \multicolumn{7}{|c|}{$\overline{Z Z=0.02 / \alpha \text {-enhanced }}$} & \multicolumn{7}{|c|}{$Z=0.008 / \alpha$-enhanced } \\
\hline$M_{\text {ZAMS }}$ & $t_{\mathrm{MS}}$ & $t_{\mathrm{RGB}}$ & $t_{\mathrm{He}-\mathrm{b}}$ & $t_{\text {EAGB }}$ & $M_{\mathrm{tot}}(1)$ & $M_{\mathrm{c}}(1)$ & $M_{\text {ZAMS }}$ & $t_{\mathrm{MS}}$ & $t_{\mathrm{RGB}}$ & $t_{\mathrm{He}-\mathrm{b}}$ & $t_{\text {EAGB }}$ & $M_{\mathrm{tot}}(1)$ & $M_{\mathrm{c}}(1)$ \\
\hline 1.0 & 8442.0 & 3065.5 & 112.02 & 10.614 & 0.713 & 0.502 & 1.0 & 6633.4 & 2419.4 & 98.966 & 10.164 & 0.755 & 0.508 \\
\hline 1.2 & 4153.6 & 1607.1 & 105.88 & 10.772 & 0.985 & 0.508 & 1.2 & 3441.4 & 1194.1 & 98.397 & 9.2639 & 1.011 & 0.517 \\
\hline 1.5 & 2287.8 & 304.00 & 99.117 & 11.415 & 1.346 & 0.514 & 1.5 & 1971.4 & 218.60 & 97.908 & 8.8516 & 1.360 & 0.526 \\
\hline 1.6 & 1865.5 & 212.48 & 109.24 & 9.9499 & 1.468 & 0.513 & 1.6 & 1619.9 & 154.04 & 100.92 & 9.3640 & 1.483 & 0.522 \\
\hline 1.8 & 1465.8 & 99.847 & 130.85 & 13.305 & 1.569 & 0.501 & 1.8 & 1147.0 & 86.083 & 118.83 & 11.511 & 1.616 & 0.505 \\
\hline 2.0 & 1075.0 & 58.364 & 177.34 & 15.423 & 1.867 & 0.488 & 2.0 & 850.65 & 35.418 & 238.79 & 15.050 & 1.939 & 0.464 \\
\hline 2.6 & 510.25 & 12.297 & 140.08 & 10.175 & 2.529 & 0.513 & 2.6 & 421.73 & 10.360 & 111.36 & 5.6708 & 2.503 & 0.578 \\
\hline 3.0 & 344.35 & 7.1561 & 83.500 & 5.7529 & 2.909 & 0.585 & 3.0 & 292.00 & 6.0452 & 62.486 & 3.2070 & 2.857 & 0.684 \\
\hline 4.0 & 146.84 & 2.7657 & 28.801 & 1.9129 & 3.823 & 0.766 & 4.0 & 144.93 & 2.3134 & 22.858 & 1.3036 & 3.811 & 0.799 \\
\hline 5.0 & 85.371 & 1.3850 & 14.328 & 0.8164 & 4.802 & 0.846 & 5.0 & 87.124 & 1.2145 & 121.36 & 0.6650 & 4.771 & 0.875 \\
\hline 6.0 & 56.370 & 0.7959 & 8.3754 & 0.4509 & 5.715 & 0.946 & 6.0 & 58.838 & 0.7307 & 7.5395 & 0.4267 & 5.713 & 0.996 \\
\hline \multicolumn{7}{|c|}{$Z=0.04 / \alpha$-enhanced } & \multicolumn{7}{|c|}{$Z=0.004 / \alpha$-enhanced } \\
\hline$M_{\text {ZAMS }}$ & $t_{\mathrm{MS}}$ & $t_{\mathrm{RGB}}$ & $t_{\mathrm{He}-\mathrm{b}}$ & $t_{\text {EAGB }}$ & $M_{\text {tot }}(1)$ & $M_{\mathrm{c}}(1)$ & $M_{\text {ZAMS }}$ & $t_{\mathrm{MS}}$ & $t_{\mathrm{RGB}}$ & $t_{\mathrm{He}-\mathrm{b}}$ & $t_{\mathrm{EAGB}}$ & $M_{\mathrm{tot}}(1)$ & $M_{\mathrm{c}}(1)$ \\
\hline 1.0 & 8445.6 & 3482.5 & 111.90 & 13.314 & 0.678 & 0.505 & 1.0 & 5696.1 & 2004.1 & 94.355 & 9.5685 & 0.784 & 0.515 \\
\hline 1.2 & 4181.9 & 1728.5 & 107.84 & 12.716 & 0.964 & 0.512 & 1.2 & 3053.4 & 974.38 & 93.588 & 8.6953 & 1.032 & 0.523 \\
\hline 1.5 & 2293.0 & 315.64 & 108.53 & 11.806 & 1.333 & 0.518 & 1.5 & 1781.6 & 173.96 & 93.097 & 8.6722 & 1.379 & 0.531 \\
\hline 1.6 & 1861.1 & 222.36 & 109.39 & 12.835 & 1.462 & 0.514 & 1.6 & 1468.9 & 124.17 & 99.306 & 8.6461 & 1.498 & 0.529 \\
\hline 1.8 & 1291.9 & 125.72 & 131.82 & 12.497 & 1.562 & 0.504 & 1.8 & 1047.6 & 67.684 & 126.58 & 10.905 & 1.662 & 0.506 \\
\hline 2.0 & 1108.6 & 54.286 & 226.81 & 17.853 & 1.855 & 0.494 & 2.0 & 782.24 & 28.666 & 197.48 & 10.426 & 1.933 & 0.494 \\
\hline 2.6 & 509.95 & 13.146 & 140.91 & 10.013 & 2.520 & 0.515 & 2.6 & 392.18 & 8.6687 & 79.021 & 3.9082 & 2.469 & 0.648 \\
\hline 3.0 & 337.90 & 7.6362 & 83.252 & 6.2347 & 2.908 & 0.574 & 3.0 & 277.07 & 5.2243 & 48.452 & 2.5145 & 2.833 & 0.744 \\
\hline 4.0 & 132.12 & 3.0023 & 32.044 & 2.3654 & 3.804 & 0.749 & 4.0 & 140.83 & 2.0926 & 21.321 & 0.9967 & 3.801 & 0.827 \\
\hline 5.0 & 74.606 & 1.4437 & 15.476 & 0.9332 & 4.753 & 0.830 & 5.0 & 86.130 & 1.1276 & 11.536 & 0.5443 & 4.786 & 0.908 \\
\hline 6.0 & 48.420 & 0.8106 & 8.8116 & 0.4817 & 5.397 & 0.935 & 6.0 & 58.868 & 0.6937 & 7.1015 & 0.3700 & 5.740 & 1.050 \\
\hline
\end{tabular}

\begin{tabular}{ccccccc}
\hline \hline \multicolumn{7}{c}{$Z=0.0005 / \alpha$-enhanced } \\
\hline$M_{\text {ZAMS }}$ & $t_{\mathrm{MS}}$ & $t_{\mathrm{RGB}}$ & $t_{\mathrm{He}-\mathrm{b}}$ & $t_{\text {EAGB }}$ & $M_{\text {tot }}(1)$ & $M_{\mathrm{c}}(1)$ \\
\hline 1.0 & 5034.3 & 1246.6 & 82.220 & 9.1452 & 0.843 & 0.527 \\
1.2 & 2650.9 & 725.59 & 80.412 & 8.3182 & 1.077 & 0.538 \\
1.5 & 1536.4 & 142.67 & 87.219 & 6.1783 & 1.409 & 0.559 \\
1.6 & 1268.5 & 104.90 & 91.057 & 5.9047 & 1.523 & 0.562 \\
1.8 & 904.79 & 47.096 & 144.84 & 6.4154 & 1.700 & 0.554 \\
2.0 & 676.61 & 22.188 & 126.53 & 5.4208 & 1.903 & 0.588 \\
2.6 & 348.59 & 7.1208 & 59.499 & 2.3104 & 2.467 & 0.737 \\
3.0 & 249.01 & 4.4098 & 38.353 & 1.7691 & 2.859 & 0.777 \\
4.0 & 131.74 & 1.8480 & 17.972 & 0.7419 & 3.871 & 0.862 \\
5.0 & 82.733 & 1.0312 & 9.8540 & 0.4491 & 4.878 & 0.943 \\
6.0 & 57.554 & 0.6858 & 6.2844 & - & - & \\
\hline
\end{tabular}


A. Weiss and J. W. Ferguson: AGB model grid, Online Material p 4

\section{Appendix B: Summary of AGB properties}

Table B.1. Same as Table 4, but for mixtures I and II (Table 1) with $Z=0.04$.

\begin{tabular}{|c|c|c|c|c|c|c|c|c|c|c|c|c|}
\hline \multirow[t]{2}{*}{$M_{\text {ZAMS }}$} & \multirow{2}{*}{$\begin{array}{l}\text { No } \\
\text { TPs }\end{array}$} & \multirow[t]{2}{*}{$M_{\mathrm{c}}(1)$} & \multirow[t]{2}{*}{$M_{\mathrm{c}}(3 \mathrm{du})$} & \multirow{2}{*}{$\begin{array}{c}\text { No } \\
\mathrm{TP}_{3 \mathrm{du}}(\mathrm{i})\end{array}$} & \multirow[t]{2}{*}{$\mathrm{C} / \mathrm{O}_{\mathrm{f}}$} & \multirow[t]{2}{*}{$M(\mathrm{f})$} & \multirow[t]{2}{*}{$M_{\mathrm{c}}(\mathrm{f})$} & \multirow{2}{*}{$\begin{array}{c}\text { No } \\
\mathrm{TP}_{\mathrm{HBB}}(\mathrm{i})\end{array}$} & \multirow{2}{*}{$\begin{array}{c}\text { No } \\
\mathrm{TP}_{\mathrm{HBB}}(\mathrm{f})\end{array}$} & \multicolumn{3}{|c|}{ TP-AGB lifetime $\left(10^{3} \mathrm{yrs}\right)$} \\
\hline & & & & & & & & & & $t_{\mathrm{mod}}$ & $\approx t_{\mathrm{e}}(\mathrm{TPs})$ & $t_{\mathrm{TP}}$ \\
\hline \multicolumn{13}{|c|}{$Z=0.04 /$ solar (mixture I) } \\
\hline 1.0 & 2 & 0.504 & - & - & 0.381 & 0.514 & 0.510 & - & - & 244.754 & - & 245 \\
\hline 1.2 & 4 & 0.513 & - & - & 0.356 & 0.524 & 0.521 & - & - & 311.523 & - & 311 \\
\hline 1.5 & 6 & 0.513 & - & - & 0.331 & 0.533 & 0.531 & - & - & 523.002 & - & 523 \\
\hline 1.6 & 7 & 0.510 & - & - & 0.334 & 0.544 & 0.536 & - & - & 705.362 & $0.5(0)$ & 706 \\
\hline 1.8 & 10 & 0.496 & - & - & 0.323 & 0.539 & 0.537 & - & - & 1107.536 & - & 1108 \\
\hline 2.0 & 13 & 0.481 & - & - & 0.322 & 0.552 & 0.552 & - & - & 1876.474 & - & 1876 \\
\hline 2.6 & 15 & 0.528 & 0.566 & 10 & 0.449 & 1.037 & 0.587 & - & - & 999.657 & $28(0)$ & 1028 \\
\hline 3.0 & 10 & 0.596 & 0.605 & 4 & 0.507 & 0.976 & 0.628 & - & - & 402.750 & $14(0)$ & 417 \\
\hline 4.0 & 8 & 0.750 & 0.751 & 2 & 0.583 & 1.988 & 0.751 & - & - & 86.755 & $31(2)$ & 118 \\
\hline 5.0 & 16 & 0.810 & 0.812 & 2 & 0.444 & 2.573 & 0.829 & - & - & 94.703 & $44(5)$ & 139 \\
\hline 6.0 & 26 & 0.893 & 0.893 & 1 & 0.455 & 3.333 & 0.908 & 6 & 17 & 74.350 & $48(12)$ & 86 \\
\hline \multicolumn{13}{|c|}{$Z=0.04 / \alpha$-enhanced (mixture II) } \\
\hline 1.0 & 2 & 0.505 & - & - & 0.163 & 0.516 & 0.510 & - & - & 235.332 & - & 235 \\
\hline 1.2 & 3 & 0.512 & - & - & 0.154 & 0.523 & 0.520 & - & - & 333.567 & - & 334 \\
\hline 1.5 & 5 & 0.518 & - & - & 0.139 & 0.539 & 0.535 & - & - & 466.447 & - & 466 \\
\hline 1.6 & 6 & 0.514 & - & - & 0.150 & 0.698 & 0.536 & - & - & 576.262 & $16(0)$ & 592 \\
\hline 1.8 & 8 & 0.504 & - & - & 0.134 & 0.538 & 0.536 & - & - & 856.256 & - & 856 \\
\hline 2.0 & 13 & 0.494 & - & - & 0.135 & 0.590 & 0.552 & - & - & 1394.989 & $4(0)$ & 1399 \\
\hline 2.6 & 16 & 0.515 & 0.558 & 10 & 0.432 & 0.745 & 0.580 & - & - & 1288.965 & $5(0)$ & 1294 \\
\hline 3.0 & 13 & 0.574 & 0.587 & 5 & 0.442 & 0.912 & 0.613 & - & - & 617.336 & $9(0)$ & 626 \\
\hline 4.0 & 8 & 0.750 & 0.749 & 1 & 0.234 & 1.795 & 0.763 & - & - & 96.381 & $26(1)$ & 122 \\
\hline 5.0 & 15 & 0.830 & 0.830 & 1 & 0.225 & 2.919 & 0.845 & - & - & 77.211 & $52(8)$ & 129 \\
\hline 6.0 & 25 & 0.935 & 0.935 & 1 & 0.219 & 3.351 & 0.946 & 4 & 18 & 54.590 & 48 (12) & 103 \\
\hline
\end{tabular}

Table B.2. Same as Table 4, but for mixtures V and VI (Table 1) with $Z=0.008$.

\begin{tabular}{|c|c|c|c|c|c|c|c|c|c|c|c|c|}
\hline \multirow[t]{2}{*}{$M_{\text {ZAMS }}$} & \multirow{2}{*}{$\begin{array}{l}\text { No } \\
\text { TPs }\end{array}$} & \multirow[t]{2}{*}{$M_{\mathrm{c}}(1)$} & \multirow[t]{2}{*}{$M_{\mathrm{c}}(3 \mathrm{du})$} & \multirow{2}{*}{$\begin{array}{c}\mathrm{No} \\
\mathrm{TP}_{3 \mathrm{du}}(\mathrm{i})\end{array}$} & \multirow[t]{2}{*}{$\mathrm{C} / \mathrm{O}_{\mathrm{f}}$} & \multirow[t]{2}{*}{$M(\mathrm{f})$} & \multirow[t]{2}{*}{$M_{\mathrm{c}}(\mathrm{f})$} & \multirow{2}{*}{$\begin{array}{c}\text { No } \\
\operatorname{TP}_{\text {HBB }}(\mathrm{i})\end{array}$} & \multirow{2}{*}{$\begin{array}{c}\text { No } \\
\operatorname{TP}_{\text {HBB }}(f)\end{array}$} & \multicolumn{3}{|c|}{ TP-AGB lifetime $\left(10^{3} \mathrm{yrs}\right)$} \\
\hline & & & & & & & & & & $t_{\mathrm{mod}}$ & $\approx t_{\mathrm{e}}$ (TPs) & $t_{\mathrm{TP}}$ \\
\hline \multicolumn{13}{|c|}{$Z=0.008 /$ solar $($ mixture $V)$} \\
\hline 1.0 & 5 & 0.513 & 0.513 & 1 & 1.170 & 0.619 & 0.531 & - & - & 698.898 & $85(0)$ & 784 \\
\hline 1.2 & 4 & 0.521 & 0.521 & 1 & 1.309 & 0.663 & 0.531 & - & - & 482.655 & $41(0)$ & 524 \\
\hline 1.5 & 5 & 0.521 & 0.524 & 2 & 2.204 & 0.538 & 0.537 & - & - & 794.177 & - & 794 \\
\hline 1.6 & 7 & 0.516 & 0.520 & 2 & 2.570 & 0.555 & 0.537 & - & - & 1029.957 & $4(0)$ & 1034 \\
\hline 1.8 & 9 & 0.497 & 0.499 & 2 & 2.004 & 1.164 & 0.526 & - & - & 1556.110 & $201(1)$ & 1757 \\
\hline 2.0 & 16 & 0.464 & 0.464 & 1 & 1.732 & 1.354 & 0.528 & - & - & 4081.799 & $260(1)$ & 4342 \\
\hline 2.6 & 10 & 0.569 & 0.569 & 1 & 2.860 & 0.758 & 0.576 & - & - & 984.120 & $6(0)$ & 990 \\
\hline 3.0 & 11 & 0.672 & 0.672 & 1 & 1.699 & 2.133 & 0.686 & - & - & 352.172 & $144(3)$ & 496 \\
\hline 4.0 & 19 & 0.801 & 0.801 & 1 & 1.358 & 3.348 & 0.816 & - & - & 178.647 & $253(25)$ & 432 \\
\hline 5.0 & 38 & 0.868 & 0.868 & 1 & 1.062 & 2.443 & 0.879 & 9 & 24 & 188.148 & $62(12)$ & 250 \\
\hline 6.0 & 46 & 0.991 & 0.991 & 1 & 0.281 & 3.498 & 0.993 & 1 & 46 & 97.485 & $79(39)$ & 176 \\
\hline \multicolumn{13}{|c|}{$Z=0.008 / \alpha$-enhanced (mixture VI) } \\
\hline 1.0 & 5 & 0.508 & 0.515 & 3 & 2.354 & 0.550 & 0.523 & - & - & 667.889 & $24(0)$ & 692 \\
\hline 1.2 & 5 & 0.517 & 0.520 & 2 & 2.434 & 0.554 & 0.531 & - & - & 641.089 & $6(0)$ & 647 \\
\hline 1.5 & 4 & 0.527 & 0.527 & 1 & 1.787 & 1.034 & 0.537 & - & - & 634.407 & $50(0)$ & 684 \\
\hline 1.6 & 5 & 0.522 & 0.522 & 1 & 1.999 & 1.173 & 0.536 & - & - & 830.354 & $63(0)$ & 893 \\
\hline 1.8 & 5 & 0.505 & 0.505 & 1 & 2.365 & 0.524 & 0.522 & - & - & 1446.966 & - & 1447 \\
\hline 2.0 & 12 & 0.464 & 0.472 & 2 & 1.916 & 1.513 & 0.498 & - & - & 3458.889 & $320(1)$ & 3779 \\
\hline 2.6 & 10 & 0.578 & 0.578 & 1 & 2.530 & 0.772 & 0.592 & - & - & 921.296 & $6(0)$ & 927 \\
\hline 3.0 & 12 & 0.684 & 0.684 & 1 & 1.526 & 2.074 & 0.698 & - & - & 367.321 & 87 (2) & 454 \\
\hline 5.0 & 32 & 0.875 & 0.875 & 1 & 0.563 & 1.824 & 0.887 & 9 & 25 & 195.952 & $37(6)$ & 233 \\
\hline 6.0 & 44 & 0.996 & 0.996 & 2 & 0.508 & 3.089 & 0.988 & 1 & 42 & 108.084 & $66(22)$ & 174 \\
\hline
\end{tabular}


A. Weiss and J. W. Ferguson: AGB model grid, Online Material p 5

Table B.3. Same as Table 4, but for mixtures VII and VIII (Table 1) with $Z=0.004$.

\begin{tabular}{|c|c|c|c|c|c|c|c|c|c|c|c|c|}
\hline \multirow[t]{2}{*}{$M_{\text {ZAMS }}$} & \multirow{2}{*}{$\begin{array}{l}\text { No } \\
\text { TPs }\end{array}$} & \multirow[t]{2}{*}{$M_{\mathrm{c}}(1)$} & \multirow[t]{2}{*}{$M_{\mathrm{c}}(3 \mathrm{du})$} & \multirow{2}{*}{$\begin{array}{c}\text { No } \\
\operatorname{TP}_{3 \mathrm{du}}(\mathrm{i})\end{array}$} & \multirow[t]{2}{*}{$\mathrm{C} / \mathrm{O}_{\mathrm{f}}$} & \multirow[t]{2}{*}{$M(\mathrm{f})$} & \multirow[t]{2}{*}{$M_{\mathrm{c}}(\mathrm{f})$} & \multirow{2}{*}{$\begin{array}{c}\text { No } \\
\operatorname{TP}_{\mathrm{HBB}}(\mathrm{i})\end{array}$} & \multirow{2}{*}{$\begin{array}{c}\text { No } \\
\operatorname{TP}_{\mathrm{HBB}}(\mathrm{f})\end{array}$} & \multicolumn{3}{|c|}{ TP-AGB lifetime $\left(10^{3} \mathrm{yrs}\right)$} \\
\hline & & & & & & & & & & $t_{\text {mod }}$ & $\approx t_{\mathrm{e}}(\mathrm{TPs})$ & $t_{\mathrm{TP}}$ \\
\hline \multicolumn{13}{|c|}{$Z=0.004 /$ solar (mixture VII) } \\
\hline 1.0 & 4 & 0.517 & 0.522 & 2 & 4.279 & 0.537 & 0.531 & - & - & 590.409 & $3(0)$ & 593 \\
\hline 1.2 & 4 & 0.525 & 0.529 & 2 & 4.305 & 0.555 & 0.536 & - & - & 530.145 & $5(0)$ & 535 \\
\hline 1.5 & 5 & 0.527 & 0.531 & 2 & 3.581 & 0.731 & 0.537 & - & - & 691.108 & $19(0)$ & 710 \\
\hline 1.6 & 7 & 0.521 & 0.525 & 2 & 3.156 & 0.537 & 0.536 & - & - & 1056.562 & - & 1057 \\
\hline 1.8 & 5 & 0.499 & 0.505 & 2 & 3.318 & 0.598 & 0.493 & - & - & 1686.695 & $33(0)$ & 1720 \\
\hline 2.0 & 14 & 0.488 & 0.495 & 2 & 3.035 & 0.529 & 0.528 & - & - & 3609.374 & - & 3610 \\
\hline 2.6 & 9 & 0.639 & 0.639 & 1 & 2.868 & 0.914 & 0.653 & - & - & 463.827 & $8(0)$ & 472 \\
\hline 3.0 & 11 & 0.744 & 0.744 & 1 & 2.235 & 1.381 & 0.746 & - & - & 200.714 & $16(0)$ & 217 \\
\hline 4.0 & 26 & 0.809 & 0.809 & 1 & 2.442 & 1.655 & 0.818 & - & - & 259.943 & $2(0)$ & 263 \\
\hline 5.0 & 35 & 0.894 & 0.894 & 1 & 1.036 & 2.787 & 0.898 & 6 & 31 & 164.360 & $75(15)$ & 239 \\
\hline 6.0 & 62 & 1.060 & 1.060 & 1 & 0.707 & 3.461 & 1.047 & 1 & 62 & 88.046 & $2(30)$ & 90 \\
\hline \multicolumn{13}{|c|}{$Z=0.004 / \alpha$-enhanced (mixture VIII) } \\
\hline 1.0 & 5 & 0.515 & 0.515 & 1 & 3.175 & 0.554 & 0.532 & - & - & 682.685 & $19(0)$ & 702 \\
\hline 1.2 & 5 & 0.523 & 0.523 & 1 & 4.177 & 0.538 & 0.533 & - & - & 674.829 & $0.6(0)$ & 675 \\
\hline 1.5 & 5 & 0.531 & 0.531 & 1 & 3.365 & 0.550 & 0.543 & - & - & 783.520 & - & 784 \\
\hline 1.6 & 3 & 0.529 & 0.529 & 1 & 2.752 & 0.521 & 0.519 & - & - & 894.331 & - & 894 \\
\hline 1.8 & 8 & 0.506 & 0.506 & 1 & 3.123 & 0.529 & 0.526 & - & - & 1529.455 & - & 1529 \\
\hline 2.0 & 10 & 0.498 & 0.498 & 2 & 3.449 & 0.519 & 0.512 & - & - & 2199.189 & $0.2(0)$ & 2200 \\
\hline 2.6 & 10 & 0.647 & 0.647 & 1 & 2.522 & 1.632 & 0.665 & - & - & 443.615 & $61(1)$ & 505 \\
\hline 5.0 & 38 & 0.908 & 0.908 & 1 & 0.772 & 2.642 & 0.912 & 4 & 32 & 159.533 & $69(13)$ & 229 \\
\hline 6.0 & 70 & 1.050 & 1.050 & 1 & 0.372 & 3.366 & 1.048 & 1 & 70 & 100.072 & $73(36)$ & 173 \\
\hline
\end{tabular}

Table B.4. Same as Table 4, but for mixtures IX and X (Table 1) with $Z=0.0005$.

\begin{tabular}{|c|c|c|c|c|c|c|c|c|c|c|c|c|}
\hline \multirow[t]{2}{*}{$M_{\text {ZAMS }}$} & \multirow{2}{*}{$\begin{array}{l}\text { No } \\
\text { TPs }\end{array}$} & \multirow[t]{2}{*}{$M_{\mathrm{c}}(1)$} & \multirow[t]{2}{*}{$M_{\mathrm{c}}(3 \mathrm{du})$} & \multirow{2}{*}{$\begin{array}{c}\text { No } \\
\mathrm{TP}_{3 \mathrm{du}}(\mathrm{i})\end{array}$} & \multirow[t]{2}{*}{$\mathrm{C} / \mathrm{O}_{\mathrm{f}}$} & \multirow[t]{2}{*}{$M(\mathrm{f})$} & \multirow[t]{2}{*}{$M_{\mathrm{c}}(\mathrm{f})$} & \multirow{2}{*}{$\begin{array}{c}\text { No } \\
\mathrm{TP}_{\mathrm{HBB}}(\mathrm{i})\end{array}$} & \multirow{2}{*}{$\begin{array}{c}\text { No } \\
\operatorname{TP}_{\mathrm{HBB}}(\mathrm{f})\end{array}$} & \multicolumn{3}{|c|}{ TP-AGB lifetime $\left(10^{3} \mathrm{yrs}\right)$} \\
\hline & & & & & & & & & & $t_{\text {mod }}$ & $\approx t_{\mathrm{e}}$ (TPs) & $t_{\mathrm{TP}}$ \\
\hline \multicolumn{13}{|c|}{$Z=0.0005 /$ solar $($ mixture IX) } \\
\hline 1.0 & 4 & 0.523 & 0.527 & 2 & 5.184 & 0.539 & 0.537 & - & - & 600.331 & - & 600 \\
\hline 1.2 & 4 & 0.537 & 0.548 & 3 & 4.125 & 0.551 & 0.550 & - & - & 518.908 & - & 519 \\
\hline 1.5 & 4 & 0.570 & 0.582 & 3 & 4.219 & 0.579 & 0.578 & - & - & 553.484 & - & 553 \\
\hline 1.6 & 5 & 0.561 & 0.566 & 2 & 4.462 & 0.579 & 0.578 & - & - & 619.811 & - & 620 \\
\hline 1.8 & 5 & 0.551 & 0.551 & 1 & 4.205 & 0.565 & 0.564 & - & - & 733.834 & - & 734 \\
\hline 2.0 & 6 & 0.584 & 0.588 & 2 & 4.180 & 0.600 & 0.599 & - & - & 603.197 & - & 603 \\
\hline 2.6 & 9 & 0.733 & 0.733 & 1 & 3.560 & 1.019 & 0.734 & - & - & 211.692 & $3(0)$ & 215 \\
\hline 3.0 & 10 & 0.780 & 0.780 & 1 & 3.776 & 1.277 & 0.772 & - & - & 162.996 & $25(1)$ & 188 \\
\hline 4.0 & 20 & 0.848 & 0.848 & 2 & 1.861 & 2.397 & 0.829 & - & - & 178.286 & $31(3)$ & 209 \\
\hline \multicolumn{13}{|c|}{$Z=0.0005 / \alpha$-enhanced (mixture X) } \\
\hline 1.0 & 3 & 0.528 & 0.528 & 1 & 6.183 & 0.535 & 0.533 & - & - & 446.329 & - & 446 \\
\hline 1.2 & 3 & 0.538 & 0.538 & 1 & 6.590 & 0.547 & 0.545 & - & - & 483.113 & - & 483 \\
\hline 1.5 & 4 & 0.560 & 0.560 & 1 & 5.602 & 0.568 & 0.567 & - & - & 510.437 & - & 510 \\
\hline 1.6 & 3 & 0.564 & 0.564 & 1 & 2.568 & 0.559 & 0.558 & - & - & 456.902 & - & 457 \\
\hline 1.8 & 6 & 0.555 & 0.555 & 1 & 4.588 & 0.561 & 0.561 & - & - & 752.815 & - & 753 \\
\hline 2.0 & 6 & 0.588 & 0.588 & 1 & 4.716 & 0.599 & 0.598 & - & - & 577.109 & - & 577 \\
\hline
\end{tabular}


A. Weiss and J. W. Ferguson: AGB model grid, Online Material p 6

\section{Appendix C: Post-AGB evolution}

Table C.1. Post-AGB evolution for all models with solar-scaled metal distributions computed through this phase ${ }^{a}$.

\begin{tabular}{|c|c|c|c|c|c|c|c|c|}
\hline$M_{\text {ZAMS }}$ & $T_{\text {eff }, \mathrm{i}}$ & $M_{\mathrm{T} 4}$ & $t_{\mathrm{tr}}$ & $t_{1}$ & $t_{\mathrm{cr}}$ & ?-burner & $\mathrm{TP}$ & End \\
\hline \multicolumn{9}{|c|}{$Z=0.0005 /$ solar } \\
\hline 1.0 & 3.68 & 0.537 & 4.274 & 5.365 & 14.464 & $\mathrm{H}$ & no & WD \\
\hline 1.2 & 3.69 & 0.550 & 2.403 & 2.868 & 8.845 & $\mathrm{H}$ & no & WD \\
\hline 1.5 & 3.72 & 0.579 & 0.905 & 1.008 & 3.441 & $\mathrm{H}$ & no & WD \\
\hline 1.6 & 3.72 & 0.578 & 1.076 & 1.213 & 4.129 & $\mathrm{H}$ & no & WD \\
\hline 1.8 & 3.71 & 0.565 & 0.545 & 0.860 & 3.917 & $\mathrm{H}$ & no & WD \\
\hline 2.0 & 3.73 & 0.600 & 0.335 & 0.478 & 2.412 & $\mathrm{H}$ & no & WD \\
\hline \multicolumn{9}{|c|}{$\mathrm{Z}=0.004 /$ solar } \\
\hline 1.0 & $3.71 *$ & 0.528 & 0.030 & $1.116^{*}$ & 16.348 & $\mathrm{H}$ & no & WD \\
\hline 1.2 & $3.71^{*}$ & 0.531 & 0.032 & $1.386^{*}$ & 16.816 & $\mathrm{H}$ & no & W \\
\hline 1.5 & $3.71 *$ & 0.536 & 0.276 & $0.681^{*}$ & 8.149 & $\mathrm{H} / \mathrm{He}$ & VLTP & $2^{\text {nd }} A G B$ \\
\hline 1.6 & 3.69 & 0.536 & 1.605 & 1.909 & 6.330 & $\mathrm{H}$ & no & WD \\
\hline 1.8 & $3.71 *$ & 0.492 & 0.020 & $1.029^{*}$ & 2.810 & $\mathrm{H} / \mathrm{He}$ & LTP & $2^{\text {nd }} \mathrm{AGB}$ \\
\hline 2.0 & 3.68 & 0.528 & 1.325 & 2.770 & 10.709 & $\mathrm{H}$ & no & WD \\
\hline \multicolumn{9}{|c|}{$Z=0.008 /$ solar } \\
\hline 1.0 & $3.70^{*}$ & 0.531 & 0.577 & $1.730 *$ & 16.935 & $\mathrm{H} / \mathrm{He}$ & VLTP & $2^{\text {nd }}$ AGB \\
\hline 1.2 & $3.71 *$ & 0.529 & 0.027 & $0.030 *$ & 14.369 & $\mathrm{H}$ & no & WD \\
\hline 1.5 & 3.69 & 0.537 & 1.840 & 2.178 & 6.995 & $\mathrm{H} / \mathrm{He}$ & VLTP & during VLTP \\
\hline 1.6 & $3.72 *$ & 0.537 & 0.192 & $0.443 *$ & 5.595 & $\mathrm{H} / \mathrm{He}$ & VLTP & $2^{\text {nd }}$ AGB \\
\hline 1.8 & $3.72 *$ & 0.526 & 0.021 & $0.025 *$ & 12.811 & $\mathrm{H}$ & no & WD \\
\hline 2.0 & $3.73^{*}$ & 0.528 & 0.013 & $0.014 *$ & 5.173 & $\mathrm{H}$ & no & WD \\
\hline \multicolumn{9}{|c|}{$Z=0.02 /$ solar } \\
\hline 1.0 & $3.71 *$ & 0.506 & 0.037 & $0.041 *$ & 26.417 & $\mathrm{H}$ & no & WD \\
\hline 1.2 & $3.70^{*}$ & 0.523 & 0.033 & $0.038 *$ & 16.099 & $\mathrm{H}$ & no & WD \\
\hline 1.5 & $3.70^{*}$ & 0.538 & 0.445 & $0.832 *$ & 7.388 & $\mathrm{H}$ & no & WD \\
\hline 1.6 & $3.72 *$ & 0.541 & 0.020 & $0.021^{*}$ & 6.248 & $\mathrm{H} / \mathrm{He}$ & VLTP & $2^{\text {nd }} A G B$ \\
\hline 1.8 & $3.73^{*}$ & 0.524 & 0.667 & $29.316^{*}$ & 47.833 & $\mathrm{H}$ & no & WD \\
\hline 2.0 & 3.70 & 0.543 & 0.528 & 0.868 & 3.614 & $\mathrm{H}$ & no & WD \\
\hline \multicolumn{9}{|c|}{$Z=0.04 /$ solar } \\
\hline 1.0 & 3.57 & 49.717 & 0.510 & 57.677 & 86.516 & $\mathrm{He}$ & no & WD \\
\hline 1.2 & 3.60 & 4.042 & 0.522 & 31.515 & 48.850 & $\mathrm{He}$ & no & WD \\
\hline 1.5 & 3.64 & 7.858 & 0.531 & 10.215 & 18.553 & $\mathrm{H}$ & no & WD \\
\hline 1.6 & $3.71 *$ & 0.021 & 0.536 & $0.029 *$ & 6.926 & $\mathrm{H}$ & no & WD \\
\hline 1.8 & $3.70^{*}$ & 0.783 & 0.536 & $1.805^{*}$ & 6.875 & $\mathrm{H}$ & no & WD \\
\hline 2.0 & 3.70 & 0.628 & 0.552 & 1.131 & 4.523 & $\mathrm{H}$ & no & WD \\
\hline
\end{tabular}

${ }^{a}$ See text for explanation of column contents; all times in $10^{3}$ years. 
A. Weiss and J. W. Ferguson: AGB model grid, Online Material $p 7$

Table C.2. As Table C.1, but for the $\alpha$-enhanced composition tracks.

\begin{tabular}{|c|c|c|c|c|c|c|c|c|}
\hline$M_{\text {ZAMS }}$ & $T_{\text {eff }, \mathrm{i}}$ & $M_{\mathrm{T} 4}$ & $t_{\mathrm{tr}}$ & $t_{1}$ & $t_{\mathrm{cr}}$ & ?-burner & $\mathrm{TP}$ & End \\
\hline \multicolumn{9}{|c|}{$Z=0.0005 / \alpha$-enhanced } \\
\hline 1.0 & 3.67 & 0.533 & 4.880 & 6.219 & 16.959 & $\mathrm{H}$ & no & WD \\
\hline 1.2 & 3.69 & 0.545 & 2.742 & 3.431 & 10.276 & $\mathrm{H}$ & no & WD \\
\hline 1.5 & 3.71 & 0.567 & 1.341 & 1.585 & 5.490 & $\mathrm{H}$ & no & WD \\
\hline 1.6 & 3.71 & 0.558 & 1.248 & 1.438 & 4.958 & $\mathrm{H} / \mathrm{He}$ & VLTP & $2^{\text {nd }} \mathrm{AGB}$ \\
\hline 1.8 & 3.71 & 0.561 & 0.575 & 1.008 & 4.649 & $\mathrm{H}$ & no & WD \\
\hline 2.0 & 3.73 & 0.598 & 0.332 & 0.510 & 3.621 & $\mathrm{H}$ & no & WD \\
\hline \multicolumn{9}{|c|}{$\mathrm{Z}=0.004 / \alpha$-enhanced } \\
\hline 1.0 & $3.71 *$ & 0.530 & 3.169 & $4.600 *$ & 17.434 & $\mathrm{H}$ & no & WD \\
\hline 1.2 & $3.72 *$ & 0.538 & 1.985 & $2.690 *$ & 11.122 & $\mathrm{H} / \mathrm{He}$ & VLTP & $2^{\text {nd }} \mathrm{AGB}$ \\
\hline 1.5 & 3.70 & 0.543 & 1.725 & 2.062 & 6.962 & $\mathrm{H}$ & no & WD \\
\hline 1.6 & 3.69 & 0.519 & 1.636 & 1.969 & 3.728 & $\mathrm{H} / \mathrm{He}$ & VLTP & $2^{\text {nd }} A G B$ \\
\hline 1.8 & 3.67 & 0.527 & 1.756 & 4.160 & 13.545 & $\mathrm{H}$ & no & WD \\
\hline 2.0 & 3.73 & 0.515 & 0.510 & 1.014 & 0.016 & $\mathrm{H} / \mathrm{He}$ & LTP & $2^{\text {nd }} A G B$ \\
\hline \multicolumn{9}{|c|}{$Z=0.008 / \alpha$-enhanced } \\
\hline 1.0 & $3.71 *$ & 0.524 & 2.572 & $3.599 *$ & 1.141 & $\mathrm{H} / \mathrm{He}$ & LTP & $2^{\text {nd }} \mathrm{AGB}$ \\
\hline 1.2 & $3.72 *$ & 0.531 & 0.027 & $0.732 *$ & 13.983 & $\mathrm{H} / \mathrm{He}$ & VLTP & $2^{\text {nd }} \mathrm{AGB}$ \\
\hline 1.5 & $3.73 *$ & 0.537 & 0.016 & $0.016^{*}$ & 0.859 & $\mathrm{H} / \mathrm{He}$ & VLTP & $2^{\text {nd }} \mathrm{AGB}$ \\
\hline 1.6 & $3.74 *$ & 0.536 & 0.013 & $0.014 *$ & 6.688 & $\mathrm{H} / \mathrm{He}$ & VLTP & $2^{\text {nd }} \mathrm{AGB}$ \\
\hline 1.8 & 3.68 & 0.523 & 1.039 & 2.062 & 8.281 & $\mathrm{H}$ & no & WD \\
\hline 2.0 & $3.73^{*}$ & 0.498 & 0.012 & $0.013^{*}$ & 6.163 & $\mathrm{H}$ & no & WD \\
\hline \multicolumn{9}{|c|}{$Z=0.02 / \alpha$-enhanced } \\
\hline 1.0 & $3.71 *$ & 0.508 & 0.046 & $2.798^{*}$ & 16.572 & $\mathrm{H} / \mathrm{He}$ & LTP & $2^{\text {nd }} \mathrm{AGB}$ \\
\hline 1.2 & $3.72 *$ & 0.519 & 0.026 & $0.027 *$ & 14.592 & $\mathrm{H}$ & no & WD \\
\hline 1.5 & $3.71 *$ & 0.537 & 1.498 & $1.833 *$ & 7.137 & $\mathrm{H}$ & no & WD \\
\hline 1.6 & 3.70 & 0.548 & 1.368 & 1.594 & 2.903 & $\mathrm{H} / \mathrm{He}$ & LTP & $2^{\text {nd }} \mathrm{AGB}$ \\
\hline 1.8 & $3.73 *$ & 0.540 & 0.495 & $0.973 *$ & 0.844 & $\mathrm{H} / \mathrm{He}$ & LTP & $2^{\text {nd }} \mathrm{AGB}$ \\
\hline 2.0 & $3.71 *$ & 0.537 & 0.113 & $0.403 *$ & 3.827 & $\mathrm{H}$ & no & WD \\
\hline \multicolumn{9}{|c|}{$Z=0.04 / \alpha$-enhanced } \\
\hline 1.0 & 3.58 & 0.511 & 51.989 & 58.338 & 35.345 & $\mathrm{He} / \mathrm{He}$ & LTP & $2^{\text {nd }} \mathrm{AGB}$ \\
\hline 1.2 & 3.63 & 0.521 & 10.657 & 14.043 & 25.677 & $\mathrm{H}$ & no & WD \\
\hline 1.5 & 3.60 & 0.536 & 23.111 & 16.080 & 0.021 & $\mathrm{He}$ & no & WD \\
\hline 1.6 & $3.72 *$ & 0.535 & 24.661 & $0.021 *$ & 7.894 & $\mathrm{H} / \mathrm{He}$ & VLTP & $2^{\text {nd }} \mathrm{AGB}$ \\
\hline 1.8 & 3.66 & 0.537 & 2.417 & 5.980 & 16.977 & $\mathrm{H}$ & no & WD \\
\hline 2.0 & $3.73^{*}$ & 0.552 & 0.539 & $0.885^{*}$ & 0.387 & $\mathrm{H} / \mathrm{He}$ & LTP & $2^{\text {nd }} A G B$ \\
\hline
\end{tabular}

